\title{
Periodicities in cluster algebras and dilogarithm identities
}

\author{
Tomoki Nakanishi
}

\begin{abstract}
We consider two kinds of periodicities of mutations in cluster algebras. For any sequence of mutations under which exchange matrices are periodic, we define the associated T- and Y-systems. When the sequence is 'regular', they are particularly natural generalizations of the known 'classic' T- and Y-systems. Furthermore, for any sequence of mutations under which seeds are periodic, we formulate the associated dilogarithm identity. We prove the identities when exchange matrices are skew symmetric.
\end{abstract}

2010 Mathematics Subject Classification. Primary 13F60; Secondary 17B37

Keywords. cluster algebras, T-systems, Y-systems, dilogarithm

\section{Introduction}

Cluster algebras were introduced by Fomin and Zelevinsky [FZ02]. They naturally appear in several different areas of mathematics, for example, in geometry of surfaces, in coordinate rings of algebraic varieties related to Lie groups, in the representation theory of algebras, and also in the representation theory of quantum groups, etc. See [FG07, GSV05, GLS09, CC06, $\mathrm{BMR}^{+}$06, DK09, to name a few.

The simplest and the most tractable class of cluster algebras are the cluster algebras with finitely many seeds. They are called the cluster algebras of finite type and play a fundamental role in many applications. Fomin and Zelevinsky classified the cluster algebras of finite type by the Dynkin diagrams in their pioneering works [FZ03a, FZ03b. They also clarified the intimate relation between cluster algebras of finite type and root systems of finite type. In particular, a remarkable periodicity property of mutations of seeds was discovered and proved; it is related to the Coxeter elements of the Weyl groups [FZ03a, FZ03b, FZ07.

In this paper we focus on two kinds of periodicities of mutations in general cluster algebras. The first one is the periodicity of exchange matrices (or quivers) under a sequence of mutations. In other words, the exchange relations of clusters and coefficient tuples are periodic under such a sequence of mutations. The second one is the periodicity of seeds under a sequence of mutations. The latter periodicity implies the former one, but the converse is not true.

Let us briefly explain the background for this study. Many examples of such periodicities appeared in connection with systems of algebraic relations called $T$ systems and Y-systems Zam91, KP92, KN92, RTV93, KNS94, Her07 and the dilogarithm identities [KR86, Kir89, BR90, KR90, Kun93, GT95] which originated in the study of integrable models in two dimensions. In retrospect, they are part of 
the cluster algebraic structure which appeared prior to the notion of cluster algebra itself. Naturally and inevitably, their cluster algebraic nature has been gradually revealed recently [FZ03b, Cha05, FZ07, Kel10a, DK09, HL10, KNS09, IIK ${ }^{+}$10c, Nak09a, Kel10b, Nak09b, IIK ${ }^{+}$10a, IIK ${ }^{+}$10b, Nak10, NT10, and it turned out that the cluster algebraic formulation and machinery are very powerful and essential to understanding their properties.

Since examples of periodicities of exchange relations and seeds are accumulating, it may be a good time to reverse the viewpoint, namely, to formulate T-systems, Y-systems, and dilogarithm identities in a more general and unified setting, starting from general cluster algebras with such periodicity properties. This is the subject of the paper.

Let us summarize the main result of the paper. For any sequence of mutations under which exchange matrices are periodic, we define the associated T- and Y-systems. When the sequence is 'regular', they are particularly natural generalizations of the known 'classic' T- and Y-systems. The definition of the term 'regular' is found in Definition 5.1 Furthermore, for any sequence of mutations under which seeds are periodic, we formulate the associated dilogarithm identity. We prove the identities when exchange matrices are skew symmetric. We expect that there will be several applications of the result in various areas related to cluster algebras.

We mention that many examples of periodicities of exchange matrices were constructed by Fordy and Marsh [FM09] and the associated T-systems were also introduced.

The relation between cluster algebras and the dilogarithm was also studied earlier by Fock and Goncharov FG09a, FG09b. In fact, Proposition 6.7 is motivated by their formulas. However, there is a subtle but important difference; that is, we use F-polynomials of [FZ07] in (6.26). See Section 6.4 for more details.

The organization of the paper is as follows. In Section 2 we introduce the basic notions for periodicities of exchange matrices and seeds. In Section 3 we present some of known examples of periodicities of exchange matrices (or quivers) and seeds, most of which are connected to 'classic' T- and Y-systems. In Section 4 we give Restriction/Extension Theorem of periodicities of seeds. After these preparations, in Section 5, for any sequence of mutations under which exchange matrices are periodic, we define the associated T- and Y-systems. Here we do not require the periodicity of seeds. Special attention is paid to the case when the sequence is regular. In Section 6 , for any sequence of mutations under which seeds are periodic, we formulate the associated dilogarithm identity. Then, we prove the identities when exchange matrices are skew symmetric in Theorem 6.1 which is the main theorem of the paper.

\section{Acknowledgments}

I thank Rei Inoue, Osamu Iyama, Bernhard Keller, Atsuo Kuniba, Roberto Tateo, and Junji Suzuki for sharing their insights in the preceding joint works. I am 
grateful to Frédéric Chapoton, Vladimir Fock, Bernhard Keller, Robert Marsh, Pierre-Guy Plamondon, and Andrei Zelevinsky for kindly communicating their works and also for useful comments. Finally, I thank Andrzej Skowronski for his kind invitation to this proceedings volume.

\section{Periodicities of exchange matrices and seeds}

2.1. Cluster algebras with coefficients. In this subsection we recall the definition of the cluster algebras with coefficients and some of their basic properties, following the convention in [FZ07] with slight change of notations and terminology. See [FZ07] for more details and information.

Fix an arbitrary semifield $\mathbb{P}$, i.e., an abelian multiplicative group endowed with a binary operation of addition $\oplus$ which is commutative, associative, and distributive with respect to the multiplication [HW90]. Let $\mathbb{Q P}$ denote the quotient field of the group ring $\mathbb{Z P}$ of $\mathbb{P}$. Let $I$ be a finite set, and let $B=\left(b_{i j}\right)_{i, j \in I}$ be a skew symmetrizable (integer) matrix; namely, there is a diagonal positive integer matrix $D$ such that ${ }^{t}(D B)=-D B$. Let $x=\left(x_{i}\right)_{i \in I}$ be an $I$-tuple of formal variables, and let $y=\left(y_{i}\right)_{i \in I}$ be an $I$-tuple of elements in $\mathbb{P}$. For the triplet $(B, x, y)$, called the initial seed, the cluster algebra $\mathcal{A}(B, x, y)$ with coefficients in $\mathbb{P}$ is defined as follows.

Let $\left(B^{\prime}, x^{\prime}, y^{\prime}\right)$ be a triplet consisting of skew symmetrizable matrix $B^{\prime}=$ $\left(b_{i j}^{\prime}\right)_{i, j \in I}$, an $I$-tuple $x^{\prime}=\left(x_{i}^{\prime}\right)_{i \in I}$ with $x_{i}^{\prime} \in \mathbb{Q P}(x)$, and an $I$-tuple $y^{\prime}=\left(y_{i}^{\prime}\right)_{i \in I}$ with $y_{i}^{\prime} \in \mathbb{P}$. For each $k \in I$, we define another triplet $\left(B^{\prime \prime}, x^{\prime \prime}, y^{\prime \prime}\right)=\mu_{k}\left(B^{\prime}, x^{\prime}, y^{\prime}\right)$, called the mutation of $\left(B^{\prime}, x^{\prime}, y^{\prime}\right)$ at $k$, as follows.

(i) Mutation of matrix.

$$
b_{i j}^{\prime \prime}= \begin{cases}-b_{i j}^{\prime} & i=k \text { or } j=k \\ b_{i j}^{\prime}+\frac{1}{2}\left(\left|b_{i k}^{\prime}\right| b_{k j}^{\prime}+b_{i k}^{\prime}\left|b_{k j}^{\prime}\right|\right) & \text { otherwise }\end{cases}
$$

(ii) Exchange relation of coefficient tuple.

$$
y_{i}^{\prime \prime}= \begin{cases}y_{k}^{\prime-1} & i=k, \\ y_{i}^{\prime} \frac{1}{\left(1 \oplus y_{k}^{\prime-1}\right)^{b_{k i}^{\prime}}} & i \neq k, \quad b_{k i}^{\prime} \geq 0 \\ y_{i}^{\prime}\left(1 \oplus y_{k}^{\prime}\right)^{-b_{k i}^{\prime}} & i \neq k, \quad b_{k i}^{\prime} \leq 0 .\end{cases}
$$

(iii) Exchange relation of cluster.

$$
x_{i}^{\prime \prime}= \begin{cases}\frac{y_{k}^{\prime} \prod_{j: b_{j k}^{\prime}>0} x_{j}^{\prime b_{j k}^{\prime}}+\prod_{j: b_{j k}^{\prime}<0} x_{j}^{\prime-b_{j k}^{\prime}}}{\left(1 \oplus y_{k}^{\prime}\right) x_{k}^{\prime}} & i=k, \\ x_{i}^{\prime} & i \neq k .\end{cases}
$$

It is easy to see that $\mu_{k}$ is an involution, namely, $\mu_{k}\left(B^{\prime \prime}, x^{\prime \prime}, y^{\prime \prime}\right)=\left(B^{\prime}, x^{\prime}, y^{\prime}\right)$. Now, starting from the initial seed $(B, x, y)$, iterate mutations and collect all the 
resulting triplets $\left(B^{\prime}, x^{\prime}, y^{\prime}\right)$. We call $\left(B^{\prime}, x^{\prime}, y^{\prime}\right)$ a seed, $y^{\prime}$ and $y_{i}^{\prime}$ a coefficient tuple and a coefficient, $x^{\prime}$ and $x_{i}^{\prime}$, a cluster and a cluster variable, respectively. The cluster algebra $\mathcal{A}(B, x, y)$ with coefficients in $\mathbb{P}$ is the $\mathbb{Z} \mathbb{P}$-subalgebra of the rational function field $\mathbb{Q P}(x)$ generated by all the cluster variables. Similarly, the coefficient group $\mathcal{G}(B, y)$ with coefficients in $\mathbb{P}$ is the multiplicative subgroup of the semifield $\mathbb{P}$ generated by all the coefficients $y_{i}^{\prime}$ together with $1 \oplus y_{i}^{\prime}$.

It is standard to identify a skew symmetric (integer) matrix $B=\left(b_{i j}\right)_{i, j \in I}$ with a quiver $Q$ without loops or 2-cycles. The set of the vertices of $Q$ is given by $I$, and we put $b_{i j}$ arrows from $i$ to $j$ if $b_{i j}>0$. The mutation $Q^{\prime \prime}=\mu_{k}\left(Q^{\prime}\right)$ of a quiver $Q^{\prime}$ is given by the following rule: For each pair of an incoming arrow $i \rightarrow k$ and an outgoing arrow $k \rightarrow j$ in $Q^{\prime}$, add a new arrow $i \rightarrow j$. Then, remove a maximal set of pairwise disjoint 2-cycles. Finally, reverse all arrows incident with $k$.

Let $\mathbb{P}_{\text {univ }}(y)$ be the universal semifield of the $I$-tuple of generators $y=\left(y_{i}\right)_{i \in I}$, namely, the semifield consisting of the subtraction-free rational functions of formal variables $y$ with usual multiplication and addition in the rational function field $\mathbb{Q}(y)$. We write $\oplus$ in $\mathbb{P}_{\text {univ }}(y)$ as + for simplicity when it is not confusing.

From now on, unless otherwise mentioned, we set the semifield $\mathbb{P}$ for $\mathcal{A}(B, x, y)$ to be $\mathbb{P}_{\text {univ }}(y)$, where $y$ is the coefficient tuple in the initial seed $(B, x, y)$.

Let $\mathbb{P}_{\text {trop }}(y)$ be the tropical semifield of $y=\left(y_{i}\right)_{i \in I}$, which is the abelian multiplicative group freely generated by $y$ endowed with the addition $\oplus$

$$
\prod_{i} y_{i}^{a_{i}} \oplus \prod_{i} y_{i}^{b_{i}}=\prod_{i} y_{i}^{\min \left(a_{i}, b_{i}\right)} .
$$

There is a canonical surjective semifield homomorphism $\pi_{\mathbf{T}}$ (the tropical evaluation) from $\mathbb{P}_{\text {univ }}(y)$ to $\mathbb{P}_{\text {trop }}(y)$ defined by $\pi_{\mathbf{T}}\left(y_{i}\right)=y_{i}$ and $\pi_{\mathbf{T}}(\alpha)=1\left(\alpha \in \mathbb{Q}_{+}\right)$. For any coefficient $y_{i}^{\prime}$ of $\mathcal{A}(B, x, y)$, let us write $\left[y_{i}^{\prime}\right]_{\mathbf{T}}:=\pi_{\mathbf{T}}\left(y_{i}^{\prime}\right)$ for simplicity. We call $\left[y_{i}^{\prime}\right]_{\mathbf{T}}$ 's the tropical coefficients (the principal coefficients in [FZ07]). They satisfy the exchange relation (2.2) by replacing $y_{i}^{\prime}$ with $\left[y_{i}^{\prime}\right]_{\mathbf{T}}$ with $\oplus$ being the addition in (2.4). We also extend this homomorphism to the homomorphism of fields $\pi_{\mathbf{T}}:\left(\mathbb{Q P}_{\text {univ }}(y)\right)(x) \rightarrow\left(\mathbb{Q P}_{\text {trop }}(y)\right)(x)$.

To each seed $\left(B^{\prime}, x^{\prime}, y^{\prime}\right)$ of $\mathcal{A}(B, x, y)$ we attach the $F$-polynomials $F_{i}^{\prime}(y) \in$ $\mathbb{Q}(y)(i \in I)$ by the specialization of $\left[x_{i}^{\prime}\right]_{\mathbf{T}}$ at $x_{j}=1(j \in I)$. It is, in fact, a polynomial in $y$ with integer coefficients due to the Laurent phenomenon [FZ07, Proposition 3.6]. For definiteness, let us take $I=\{1, \ldots, n\}$. Then, $x^{\prime}$ and $y^{\prime}$ have the following factorized expressions [FZ07, Proposition 3.13, Corollary 6.3] by the $F$-polynomials.

$$
\begin{aligned}
& x_{i}^{\prime}=\left(\prod_{j=1}^{n} x_{j}^{g_{j i}^{\prime}}\right) \frac{F_{i}^{\prime}\left(\hat{y}_{1}, \ldots, \hat{y}_{n}\right)}{F_{i}^{\prime}\left(y_{1}, \ldots, y_{n}\right)}, \quad \hat{y}_{i}=y_{i} \prod_{j=1}^{n} x_{j}^{b_{j i}}, \\
& y_{i}^{\prime}=\left[y_{i}^{\prime}\right]_{\mathbf{T}} \prod_{j=1}^{n} F_{j}^{\prime}\left(y_{1}, \ldots, y_{n}\right)^{b_{j i}^{\prime}} .
\end{aligned}
$$

The integer vector $\mathbf{g}_{i}^{\prime}=\left(g_{1 i}^{\prime}, \ldots, g_{n i}^{\prime}\right)(i=1, \ldots, n)$ uniquely determined by (2.5) for each $x_{i}^{\prime}$ is called the $g$-vector for $x_{i}^{\prime}$. 
Conjecture 2.1 ([FZ07]). For any cluster algebra $\mathcal{A}(B, x, y)$ with skew symmetrizable matrix $B$, the following properties hold.

(a) Each tropical coefficient $\left[y_{i}^{\prime}\right]_{\mathbf{T}}$ is not 1 , and, either positive or negative Laurent monomial in $y$.

(b) (the 'sign coherence') For each $i$, the ith components of $g$-vectors, $g_{i j}^{\prime}(j=$ $1, \ldots, n+1)$ in (2.5), are simultaneously nonpositive or nonnegative.

(c) Each $F$-polynomial $F_{i}^{\prime}(y)$ has a constant term 1.

This conjecture was proved in the skew symmetric case by DWZ10, Pla10a, Nag10 with the result of [FZ07. Proposition 5.6].

Theorem 2.2. Conjecture 2.1 is true for any skew symmetric matrix B.

Let $\mathbf{i}=\left(i_{1}, \ldots, i_{r}\right)$ be an $I$-sequence, namely, $i_{1}, \ldots, i_{r} \in I$. We define the composite mutation $\mu_{\mathbf{i}}$ by $\mu_{\mathbf{i}}=\mu_{i_{r}} \cdots \mu_{i_{2}} \mu_{i_{1}}$, where the product means the composition. For $I$-sequences $\mathbf{i}$ and $\mathbf{i}^{\prime}$, we write $\mathbf{i} \sim_{B} \mathbf{i}^{\prime}$ if $\mu_{\mathbf{i}}(B, x, y)=\mu_{\mathbf{i}^{\prime}}(B, x, y)$.

The following fact will be used implicitly and frequently.

Lemma 2.3. Let $B=\left(b_{i j}\right)_{i, j \in I}$ be a skew symmetrizable matrix and let $\mathbf{i}=$ $\left(i_{1}, \ldots, i_{r}\right)$ be an $I$-sequence. Suppose that $b_{i_{a} i_{b}}=0$ for any $1 \leq a, b \leq r$. Then, the following facts hold.

(a) For any permutation $\sigma$ of $\{1, \ldots, r\}$, we have

$$
\mathbf{i} \sim_{B}\left(i_{\sigma(1)}, \ldots, i_{\sigma(r)}\right) .
$$

(b) Let $B^{\prime}=\mu_{\mathbf{i}}(B)$. Then, $b_{i_{a} i_{b}}^{\prime}=0$ holds for any $1 \leq a, b \leq r$.

(c) Let $\left(B^{\prime}, x^{\prime}, y^{\prime}\right)=\mu_{\mathbf{i}}(B, x, y)$. Then, $(B, x, y)=\mu_{\mathbf{i}}\left(B^{\prime}, x^{\prime}, y^{\prime}\right)$.

Proof. The facts (a) and (b) are easily verified from (2.1)-(2.3). The fact (c) follows from (a), (b), and the involution property of each mutation $\mu_{i}$.

\subsection{Periodicities of exchange matrices and seeds.}

Definition 2.4. Let $\mathcal{A}(B, x, y)$ be a cluster algebra, $\left(B^{\prime}, x^{\prime}, y^{\prime}\right)$ be a seed of $\mathcal{A}(B, x, y), \mathbf{i}=\left(i_{1}, \ldots, i_{r}\right)$ be an $I$-sequence, $\left(B^{\prime \prime}, x^{\prime \prime}, y^{\prime \prime}\right)=\mu_{\mathbf{i}}\left(B^{\prime}, x^{\prime}, y^{\prime}\right)$, and $\nu: I \rightarrow I$ be a bijection.

(a) We call the sequence $\mathbf{i}$ a $\nu$-period of $B^{\prime}$ if $b_{\nu(i) \nu(j)}^{\prime \prime}=b_{i j}^{\prime}(i, j \in I)$ holds; furthermore, if $\nu=\mathrm{id}$, we simply call it a period of $B^{\prime}$.

(b) We call the sequence $\mathbf{i}$ a $\nu$-period of $\left(B^{\prime}, x^{\prime}, y^{\prime}\right)$ if

$$
b_{\nu(i) \nu(j)}^{\prime \prime}=b_{i j}^{\prime}, \quad x_{\nu(i)}^{\prime \prime}=x_{i}^{\prime}, \quad y_{\nu(i)}^{\prime \prime}=y_{i}^{\prime} \quad(i, j \in I)
$$

holds; furthermore, if $\nu=$ id, we simply call it a period of $\left(B^{\prime}, x^{\prime}, y^{\prime}\right)$.

For any seed $\left(B^{\prime}, x^{\prime}, y^{\prime}\right)$ of $\mathcal{A}(B, x, y), \mathcal{A}\left(B^{\prime}, x^{\prime}, y^{\prime}\right)$ is isomorphic to $\mathcal{A}(B, x, y)$ as a cluster algebra. Therefore, by resetting the initial seed if necessary, we may concentrate on the situation where $\left(B^{\prime}, x^{\prime}, y^{\prime}\right)=(B, x, y)$ in the above without losing generality. 
If $\mathbf{i}$ is a $\nu$-period of $(B, x, y)$, then, of course, it is a $\nu$-period of $B$. However, the converse does not hold, in general.

If $\mathbf{i}$ is a $\nu$-period of $B$ and there is a nontrivial automorphism $\omega: I \rightarrow I$ of $B$, i.e., $b_{\omega(i) \omega(j)}=b_{i j}$, then $\mathbf{i}$ is also an $\nu \omega$-period. On the other hand, there is no such ambiguity for a $\nu$-period of $(B, x, y)$, since cluster variables $x_{i}(i \in I)$ are algebraically independent.

If $\mathbf{i}$ and $\mathbf{i}^{\prime}$ are a $\nu$-period and a $\nu^{\prime}$-period of $B$, or $(B, x, y)$, respectively, then the concatenation of sequences

$$
\mathbf{i} \mid \nu\left(\mathbf{i}^{\prime}\right):=\left(i_{1}, \ldots, i_{r}, \nu\left(i_{1}^{\prime}\right), \ldots, \nu\left(i_{r^{\prime}}^{\prime}\right)\right)
$$

is a $\nu \nu^{\prime}$-period of $B$, or $(B, x, y)$. In particular, $\mathbf{i}|\nu(\mathbf{i})| \cdots \mid \nu^{p-1}(\mathbf{i})$ is a $\nu^{p}$-period of $B$, or $(B, x, y)$, for any positive integer $p$. Since $\nu$ acts on a finite set $I$, it has a finite order, say, $g$. Define

$$
\mathbf{j}(\mathbf{i}, \nu):=\mathbf{i}|\nu(\mathbf{i})| \cdots \mid \nu^{g-1}(\mathbf{i}) .
$$

Then, $\mathbf{j}(\mathbf{i}, \nu)$ is a period of $B$, or $(B, x, y)$.

Examples of periodicities of exchange matrices (or quivers) and seeds will be given in Section 3

Proposition 2.5. If $\mathbf{i}$ is a period of $(B, x, y)$ in $\mathcal{A}(B, x, y)$, then $\mathbf{i}$ is also a period of $(-B, x, y)$ in $\mathcal{A}(-B, x, y)$.

Proof. This is due to the duality between the exchange $B \leftrightarrow-B, y \leftrightarrow y^{-1}$. Namely, the correspondence of seeds $\left(B^{\prime}, x^{\prime}, y^{\prime}\right)$ in $\mathcal{A}(B, x, y)$ and $\left(-B^{\prime}, x^{\prime}, y^{\prime-1}\right)$ in $\mathcal{A}\left(-B, x, y^{-1}\right)$ commutes with mutations and yields the isomorphism of cluster algebras.

When $B$ is skew symmetric, the transformation $B \leftrightarrow-B$ corresponds to the transformation $Q \leftrightarrow Q^{\text {op }}$, where $Q^{\text {op }}$ is the opposite quiver of $Q$.

2.3. Criterion of periodicity of seeds for skew symmetric case. In general, checking the condition (2.8) directly is a very difficult task. However, at least when $B$ is skew symmetric, one can reduce the condition drastically, thanks to the existence of the categorification with 2-Calabi-Yau property by Plamondon Pla10b, Pla10a.

Theorem 2.6 ([Pla10b, Pla10a], IIK ${ }^{+} 10 \mathrm{a}$, Theorem 5.1]). Assume that the matrix $B$ in Definition 2.4 is skew symmetric. Then, the condition (2.8) holds if and only if the following condition holds:

$$
\left[y_{\nu(i)}^{\prime \prime}\right]_{\mathbf{T}}=\left[y_{i}^{\prime}\right]_{\mathbf{T}} \quad(i \in I) .
$$

We expect that Theorem 2.6 holds for any skew symmetrizable matrix $B$.

Let us also mention that the application software by Bernhard Keller [Kelb] is a practical and versatile tool to check and explore periodicities of quivers and seeds. 


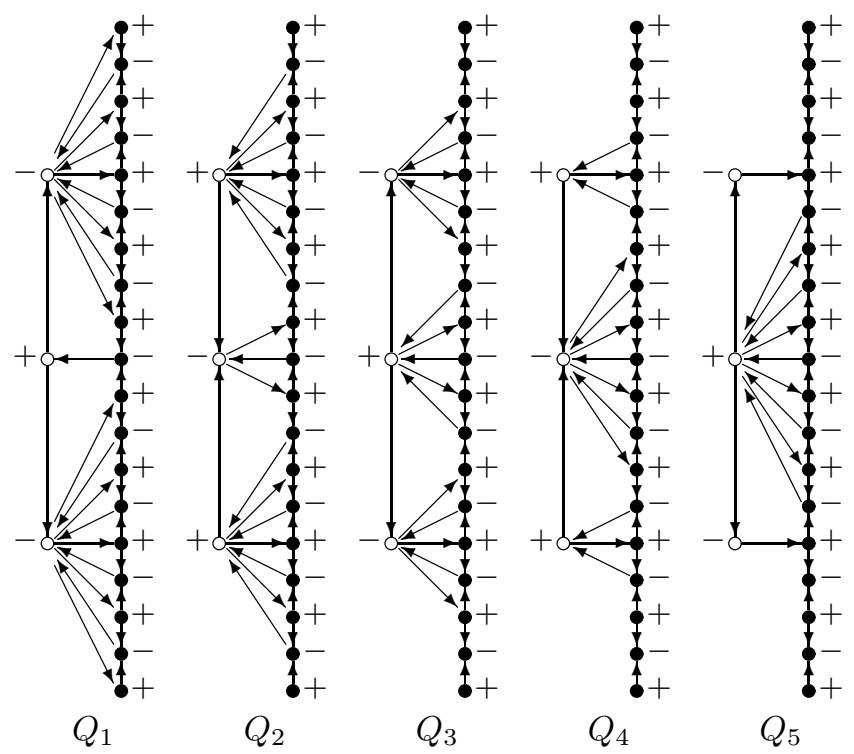

Figure 1. An example of periodicity of quivers, where we identify the right columns in all the quivers $Q_{1}, \ldots, Q_{5}$.

\section{Examples}

We present some of known examples of periodicities of exchange matrices (or quivers) and seeds.

3.1. Examples of periodicities of exchange matrices. There are plenty of examples of periodicities of exchange matrices.

We identify a skew symmetric matrix $B$ and the corresponding quiver $Q$ as in Section 2.1.

Example 3.1. Cluster algebras for bipartite matrices with alternating property [FZ07].

Assume that a skew symmetrizable matrix $B$ is bipartite; namely, the index set $I$ of $B$ admits the decomposition $I=I_{+} \sqcup I_{-}$such that, for any pair $(i, j)$ with $b_{i j} \neq 0$, either $i \in I_{+}, j \in I_{-}$or $i \in I_{-}, j \in I_{+}$holds. Assume further that $B$ has the following 'alternating' property: $b_{i j}>0$ only if $i \in I_{+}, j \in I_{-}$. (In the quiver picture, $i \in I_{+}$is a source and $i \in I_{-}$is a sink so that the quiver is alternating.) Let $\mathbf{i}_{+}$and $\mathbf{i}_{-}$be the sequences of all the distinct elements of $I_{+}$and $I_{-}$, respectively, where the order of the sequence is chosen arbitrarily thanks to Lemma 2.3. Then, $\mu_{\mathbf{i}_{+}}(B)=-B$ and $\mu_{\mathbf{i}_{-}}(-B)=B$. Thus, $\mathbf{i}=\mathbf{i}_{+} \mid \mathbf{i}_{-}$is a period of $B$. The associated Y-system and 'T-system' (in our terminology) were studied in detail in [FZ07]. (A matrix $B$ here is called a 'bipartite matrix' in [FZ07].) 
Example 3.2. Cluster algebras for $T$ - and $Y$-systems for quantum affinizations of tamely laced quantum Kac-Moody algebras [Her07, KNS09, Nak10].

The cluster algebras for T- and Y-systems for quantum affinizations of tamely laced quantum Kac-Moody algebras provide a rich family of more complicated periodicities of exchange matrices. As a typical example, let $Q$ be the quiver in Figure 1, where the right columns in the five quivers $Q_{1}, \ldots, Q_{5}$ are identified. We remark that $Q$ is not bipartite in the sense of Example 3.1. Let $\mathbf{i}_{+}^{\bullet}$ (resp. $\mathbf{i}_{-}^{\bullet}$ ) be the sequence of all the distinct elements of the vertices in $Q$ with property $(\bullet,+)$ (resp. $(\bullet,-)$ ), and let $\mathbf{i}_{+, k}^{\circ}\left(\right.$ resp. $\left.\mathbf{i}_{-, k}^{\circ}\right)$ be a sequence of all the distinct elements of the vertices in $Q_{k}$ with property $(\circ,+)$ (resp. $\left.(\circ,-)\right)$, where the order of the sequence is chosen arbitrarily thanks to Lemma 2.3, Let

$$
\begin{aligned}
\mathbf{i}= & \mathbf{i}_{+}^{\bullet}\left|\mathbf{i}_{+, 1}^{\circ}\right| \mathbf{i}_{-}^{\bullet}\left|\mathbf{i}_{+, 4}^{\circ}\right| \mathbf{i}_{+}^{\bullet}\left|\mathbf{i}_{+, 3}^{\circ}\right| \mathbf{i}_{-}^{\bullet}\left|\mathbf{i}_{+, 2}^{\circ}\right| \mathbf{i}_{+}^{\bullet} \mid \mathbf{i}_{+, 5}^{\circ} \\
& \left|\mathbf{i}_{-}^{\bullet}\right| \mathbf{i}_{-, 1}^{\circ}\left|\mathbf{i}_{+}^{\bullet}\right| \mathbf{i}_{-, 4}^{\circ}\left|\mathbf{i}_{-}^{\bullet}\right| \mathbf{i}_{-, 3}^{\circ}\left|\mathbf{i}_{+}^{\bullet}\right| \mathbf{i}_{-, 2}^{\circ}\left|\mathbf{i}_{-}^{\bullet}\right| \mathbf{i}_{-, 5}^{\circ} .
\end{aligned}
$$

Let $\sigma$ be the permutation of $\{1, \ldots, 5\}$,

$$
\sigma=\left(\begin{array}{lllll}
1 & 2 & 3 & 4 & 5 \\
3 & 1 & 5 & 2 & 4
\end{array}\right) .
$$

Let $\nu: I \rightarrow I$ be the bijection of order 5 such that each vertex in $Q_{i}$ maps to the vertex in $Q_{\sigma(i)}$ in the same position. In particular, every vertex with $\bullet$ is a fixed point of $\nu$, and every vertex with $\circ$ has the $\nu$-orbit of length 5 . Then $\mathbf{i}_{+}^{\bullet}\left|\mathbf{i}_{+, 1}^{\circ}\right| \mathbf{i}_{-}^{\bullet} \mid \mathbf{i}_{+, 4}^{\circ}$ is a $\nu$-period of $Q$, and $\mathbf{i}$ is a period of $Q$. Note that $\mathbf{i} \sim_{Q}$ $\mathbf{j}\left(\mathbf{i}_{+}^{\bullet}\left|\mathbf{i}_{+, 1}^{\circ}\right| \mathbf{i}_{-}^{\bullet} \mid \mathbf{i}_{+, 4}^{\circ}, \nu\right)$.

The quiver $Q$ corresponds to the level $\ell=4 \mathrm{~T}$ - and $\mathrm{Y}$-systems for the quantum affinization of the quantum Kac-Moody algebra whose Cartan matrix is represented by the following Dynkin diagram.

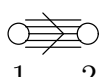

See Nak10 for more details.

Example 3.3. Cluster algebras with $\rho^{m}$-period of $Q$ for cyclic permutation $\rho$ [FM09].

For a cyclic permutation $\rho$ of $I$, many examples of quivers with $\rho^{m}$-period were constructed and partially classified in [FM09]. For instance, let $Q$ be the following quiver:

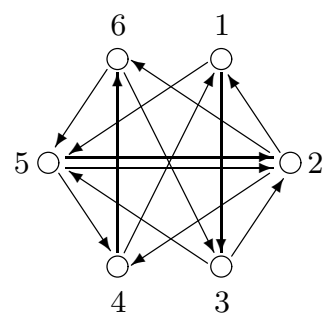


Let

$$
\mathbf{i}=(1,2,3,4,5,6) .
$$

Let $\rho$ be the cyclic permutation of $\{1, \ldots, 6\}$,

$$
\rho=\left(\begin{array}{llllll}
1 & 2 & 3 & 4 & 5 & 6 \\
2 & 3 & 4 & 5 & 6 & 1
\end{array}\right)
$$

Then, $(1,2)$ is a $\rho^{2}$-period of $Q$, and $\mathbf{i}$ is a period of $Q$. Note that $\mathbf{i}=\mathbf{j}\left((1,2), \rho^{2}\right)$.

The quiver $Q$ is a special case of a family of 'period 2 solutions' of [FM09. Section 7.4] with $m_{1}=m_{3}=-m_{2}=1$ and $m_{\overline{1}}=0$ therein. It is also the quiver for the quiver gauge theory on the del Pezzo 3 surface [FHH01]. See [FM09] for more details.

3.2. Examples of periodicities of seeds. All the examples of periodicities of seeds below can be proved by verifying the condition (2.11) in Theorem 2.6, case by case, with the help of relevant Coxeter elements.

Example 3.4. Cluster algebras of finite type [FZ03a, FZ03b.

For a skew symmetrizable matrix $B$, define a matrix $C=C(B)$ by

$$
C_{i j}= \begin{cases}2 & i=j \\ -\left|b_{i j}\right| & i \neq j .\end{cases}
$$

Then, it is known that the cluster algebra $\mathcal{A}(B, x, y)$ is of finite type if and only if $B$ is mutation equivalent to a skew-symmetric matrix $B^{\prime}$ such that $C\left(B^{\prime}\right)$ is a direct sum of Cartan matrices of finite type. Suppose that $C(B)$ is a Cartan matrix of finite type. Since there are only a finite number of seeds of $\mathcal{A}(B, x, y)$, for any $I$-sequence $\mathbf{i}$, there is some $p$ such that the $p$-fold concatenation $\mathbf{i}^{p}$ of $\mathbf{i}$ is a period of $\mathcal{A}(B, x, y)$. Among them there is some distinguished period of $(B, x, y)$, which is closely related to the Coxeter element of the Weyl group for $C$. For type $A_{n}$, for example, they are given as follows.

(a) Type $A_{n}(n$ : odd). For odd $n$, let $Q$ be the following alternating quiver with index set $I=\{1, \ldots, n\}$ :

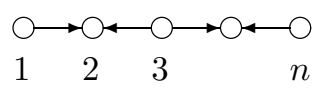

Let $\mathbf{i}=\mathbf{i}_{+} \mid \mathbf{i}_{-}$, where $\mathbf{i}_{+}=(1,3, \ldots, n), \mathbf{i}_{-}=(2,4, \ldots, n-1)$. Let $\omega: I \rightarrow I$ be the left-right reflection, which is a quiver automorphism of $Q$. Then $\mathbf{i}$ is a period of $Q$. Furthermore, $\mathbf{i}^{(n+3) / 2}$ is an $\omega$-period of $(Q, x, y)$, and $\mathbf{i}^{n+3}$ is a period of $(Q, x, y)$. We note that $n+3=h\left(A_{n}\right)+2$, where $h(X)$ is the Coxeter number of type $X$. For simply laced $X, h(X)$ coincides with the dual Coxeter number $h^{\vee}(X)$ of type $X$.

(b) Type $A_{n}(n$ : even). For even $n$, let $Q$ be the following quiver with index set $I=\{1, \ldots, n\}$ : 


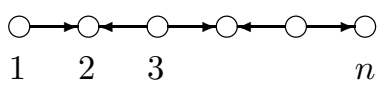

Let $\mathbf{i}=\mathbf{i}_{+} \mid \mathbf{i}_{-}$, where $\mathbf{i}_{+}=(1,3, \ldots, n-1), \mathbf{i}_{-}=(2,4, \ldots, n)$. Let $\nu: I \rightarrow I$ be the left-right reflection, which is not a quiver automorphism of $Q$. Then $\mathbf{i}_{+}$is an $\nu$ period of $Q$ and $\mathbf{i}$ is a period of $Q$. Furthermore, $\mathbf{i}^{n / 2+1} \mid \mathbf{i}_{+}$is a $\nu$-period of $(Q, x, y)$, and $\mathbf{i}^{n+3}$ is a period of $(Q, x, y)$. Note that $\mathbf{i} \sim_{Q} \mathbf{j}\left(\mathbf{i}_{+}, \nu\right)$ and $\mathbf{i}^{n+3} \sim_{Q} \mathbf{j}\left(\mathbf{i}^{n / 2+1} \mathbf{i}_{+}, \nu\right)$.

Example 3.5. Cluster algebras for $T$ - and $Y$-systems of quantum affine algebras Kel10a, Kel10b, DK09, IIK ${ }^{+} 10 \mathrm{a}$, IIK $^{+} 10 \mathrm{~b}$, IIK $^{+} 10 \mathrm{c}$.

With each pair $(X, \ell)$ of a Dynkin diagram $X$ of finite type and an integer $\ell \geq 2$, one can associate a quiver $Q=Q(X, \ell)$. They are related to the $\mathrm{T}$ - and Y-systems of a quantum affine algebra of type $X$, and provide a family of periodicities of seeds. Let us give typical examples for simply laced and nonsimply laced ones.

(a) Simply laced case: $(X, \ell)=\left(A_{4}, 4\right)$. Let $Q$ be the following quiver with index set $I$ :

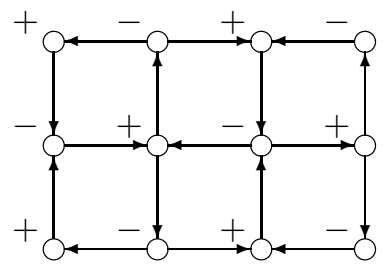

Let $\mathbf{i}_{+}$and $\mathbf{i}_{-}$be as before. Let $\mathbf{i}=\mathbf{i}_{+} \mid \mathbf{i}_{-}$. Let $\nu: I \rightarrow I$ be the left-right reflection, and let $\omega: I \rightarrow I$ be the top-bottom reflection, so that $\omega \nu=\nu \omega$. Then $\mathbf{i}_{+}$is a $\nu$-period of $Q$, and $\mathbf{i}$ is a period of $Q$. Furthermore, $\mathbf{i}^{4} \mid \mathbf{i}_{+}$is a $\nu \omega$-period of $(Q, x, y)$, and $\mathbf{i}^{9}$ is a period of $(Q, x, y)$, where $9=5+4=h\left(A_{4}\right)+\ell$. Note that $\mathbf{i} \sim_{Q} \mathbf{j}\left(\mathbf{i}_{+}, \nu\right)$ and $\mathbf{i}^{9} \sim_{Q} \mathbf{j}\left(\mathbf{i}^{4} \mathbf{i}_{+}, \nu \omega\right)$.

(b) Nonsimply laced case: $(X, \ell)=\left(B_{4}, 4\right)$. Let $Q$ be the following quiver with index set $I$ :

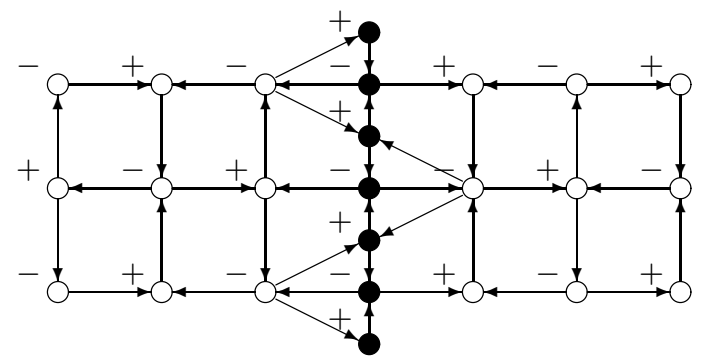

Let $\mathbf{i}_{+}^{\bullet}$ (resp. $\mathbf{i}_{-}^{\bullet}, \mathbf{i}_{+}^{\circ}, \mathbf{i}_{-}^{\circ}$ ) be a sequence of all the distinct elements of $I$ with property $(\bullet,+)$ (resp. $(\bullet,-),(\circ,+),(\circ,-))$, where the order of the sequence is chosen arbitrarily. Let

$$
\mathbf{i}=\left(\mathbf{i}_{+}^{\bullet}\left|\mathbf{i}_{+}^{\circ}\right| \mathbf{i}_{-}^{\bullet}\right) \mid\left(\mathbf{i}_{+}^{\bullet}\left|\mathbf{i}_{-}^{\circ}\right| \mathbf{i}_{-}^{\bullet}\right)
$$


Let $\nu: I \rightarrow I$ be the left-right reflection, and let $\omega: I \rightarrow I$ be the top-bottom reflection, so that $\omega \nu=\nu \omega$. Then $\mathbf{i}_{+}^{\bullet}\left|\mathbf{i}_{+}^{\circ}\right| \mathbf{i}_{-}^{\bullet}$ is a $\nu$-period of $Q$, and $\mathbf{i}$ is a period of $Q$. Furthermore, $\mathbf{i}^{5} \mid\left(\mathbf{i}_{+}^{\bullet}\left|\mathbf{i}_{+}^{\circ}\right| \mathbf{i}_{-}^{\bullet}\right)$ is a $\nu \omega$-period of $(Q, x, y)$, and $\mathbf{i}^{11}$ is a period of $(Q, x, y)$, where $11=7+4=h^{\vee}\left(B_{4}\right)+\ell$. Note that $\mathbf{i} \sim_{Q} \mathbf{j}\left(\mathbf{i}_{+}^{\bullet}\left|\mathbf{i}_{+}^{\circ}\right| \mathbf{i}_{-}^{\bullet}, \nu\right)$ and $\mathbf{i}^{11} \sim_{Q} \mathbf{j}\left(\mathbf{i}^{5} \mid\left(\mathbf{i}_{+}^{\bullet}\left|\mathbf{i}_{+}^{\circ}\right| \mathbf{i}_{-}^{\bullet}\right), \nu \omega\right)$. See $\left[\mathrm{IIK}{ }^{+} 10 \mathrm{a}\right]$ for more details.

Example 3.6. Cluster algebras for sine-Gordon T-and Y-systems [NT10].

The $\mathrm{T}$ - and Y-systems which originated from the sine-Gordon model provide another family of periodicities of seeds. Let us give an example.

Let $Q$ be the following quiver with index set $I=\{1, \ldots, 13\}$. Here all the vertices with $\bullet$ in the same position in the quivers $Q_{1}, \ldots, Q_{6}$ are identified. The quiver $Q$ is mutation equivalent to the quiver of type $D_{13}$.

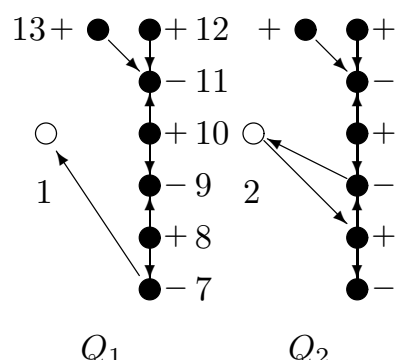

$Q_{1}$

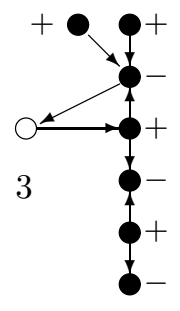

$Q_{3}$

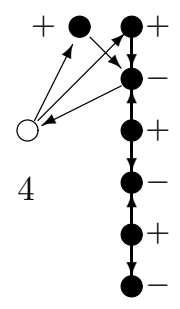

$Q_{4}$

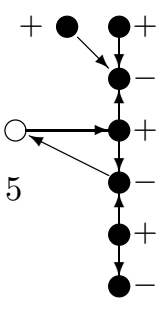

$Q_{5}$

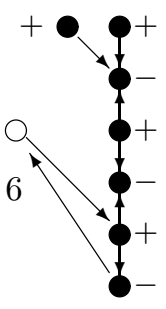

$Q_{6}$

Let $\mathbf{i}_{+}^{\bullet}$ and $\mathbf{i}_{-}^{\bullet}$ be the $I$-sequences as before. Let

$$
\mathbf{i}=\left(\mathbf{i}_{+}^{\bullet}|(1)| \mathbf{i}_{-}^{\bullet}\right)\left|\left(\mathbf{i}_{+}^{\bullet}|(2)| \mathbf{i}_{-}^{\bullet}\right)\right| \cdots \mid\left(\mathbf{i}_{+}^{\bullet}|(6)| \mathbf{i}_{-}^{\bullet}\right) .
$$

Let $\nu: I \rightarrow I$ be the bijection of order 6 cyclically mapping the vertices $1,2, \ldots$, 6 to $2,3, \ldots, 1$ and fixing the rest. Let $\omega: I \rightarrow I$ be the involution exchanging the top two vertices with $\bullet$ and fixing the rest, which is a quiver automorphism of $Q$. Note that $\omega \nu=\nu \omega$. Then $\mathbf{i}_{+}^{\bullet}|(1)| \mathbf{i}_{-}^{\bullet}$ is a $\nu$-period of $Q$, and $\mathbf{i}$ is a period of $Q$. Furthermore, $\mathbf{i}^{2} \mid\left(\mathbf{i}_{+}^{\bullet}|(1)| \mathbf{i}_{-}^{\bullet}\right)$ is a $\nu \omega$-period of $(Q, x, y)$, and $\mathbf{i}^{13}$ is a period of $(Q, x, y)$, where $13=(12+2+10+2) / 2=\left(h\left(D_{7}\right)+2+h\left(D_{6}\right)+2\right) / 2$. Note that $\mathbf{i} \sim_{Q} \mathbf{j}\left(\mathbf{i}_{+}^{\bullet}|(1)| \mathbf{i}_{-}^{\bullet}, \nu\right)$ and $\mathbf{i}^{13} \sim_{Q} \mathbf{j}\left(\mathbf{i}^{2} \mid\left(\mathbf{i}_{+}^{\bullet}|(1)| \mathbf{i}_{-}^{\bullet}\right), \nu \omega\right)$. See [NT10] for more details.

In summary, we make two plain observations in these examples.

(a) There are a variety of patterns of periodicities of seeds.

(b) It is hard to tell whether a repetition of a given period of exchange matrices yields a period of seeds just by looking at the shape of its quiver.

We expect that these examples are just a tip of iceberg of the whole class of periodicities of seeds and their classification would be very challenging but interesting. 


\section{Restriction/Extension Theorem}

In this section we present Restriction/Extension Theorem on periodicities of seeds.

We first state a very general theorem on the relation between $g$-vectors and tropical coefficients.

Let $I=\{1, \ldots, n\}$. For a given seed $\left(B^{\prime}, x^{\prime}, y^{\prime}\right)$ of $\mathcal{A}(B, x, y)$, let $\mathbf{g}_{i}^{\prime}=\left(g_{1 i}^{\prime}, \ldots, g_{n i}^{\prime}\right)$ $(i \in I)$ be the $g$-vectors defined by (2.5). We also introduce integers $c_{i j}^{\prime}(i, j=$ $1, \ldots, n)$ by

$$
\left[y_{i}^{\prime}\right]_{\mathbf{T}}=\prod_{j=1}^{n} y_{j}^{c_{j i}^{\prime}} .
$$

Consider the matrices $C^{\prime}=\left(c_{i j}^{\prime}\right)_{i, j=1}^{n}$ and $G^{\prime}=\left(g_{i j}^{\prime}\right)_{i, j=1}^{n}$; that is, each column of $C^{\prime}$ is the exponents of a tropical coefficient, and each column of $G^{\prime}$ is a $g$-vector. We remark that $C^{\prime}$ is the bottom part of the extended exchange matrix $\tilde{B}^{\prime}$ with the principal coefficients in [FZ07, Eq. (2.14)].

Theorem 4.1. Assume that $B$ is skew symmetric. Then, the matrices $C^{\prime T}$ and $G^{\prime}$ are inverse to each other, where $C^{T^{T}}$ is the transpose of $C^{\prime}$.

Proof. We prove it by the induction on mutations. When $\left(B^{\prime}, x^{\prime}, y^{\prime}\right)=(B, x, y)$, the claim holds because $C^{\prime}=G^{\prime}=I$. Suppose that $\left(B^{\prime \prime}, x^{\prime \prime}, y^{\prime \prime}\right)=\mu_{k}\left(B^{\prime}, x^{\prime}, y^{\prime}\right)$. Let $C^{\prime \prime}$ and $G^{\prime \prime}$ be the corresponding matrices. Then, the following recursion relations hold [FZ07, Eq.(5.9) \& Proposition 6.6]

$$
\begin{aligned}
& c_{i j}^{\prime \prime}= \begin{cases}-c_{i k}^{\prime} & j=k \\
c_{i j}^{\prime}+\frac{1}{2}\left(\left|c_{i k}^{\prime}\right| b_{k j}^{\prime}+c_{i k}^{\prime}\left|b_{k j}^{\prime}\right|\right) & j \neq k,\end{cases} \\
& g_{i j}^{\prime \prime}= \begin{cases}-g_{i k}^{\prime}+\sum_{p=1}^{n} g_{i p}^{\prime}\left[b_{p k}^{\prime}\right]_{+}-\sum_{p=1}^{n} b_{i p}\left[c_{p k}^{\prime}\right]_{+} & j=k, \\
g_{i j}^{\prime} & j \neq k,\end{cases}
\end{aligned}
$$

where $[a]_{+}=a$ if $a>0$ and 0 otherwise. Also the following relation holds [FZ07, Eq.(6.14)]

$$
\sum_{p=1}^{n} g_{i p}^{\prime} b_{p j}^{\prime}=\sum_{p=1}^{n} b_{i p} c_{p j}^{\prime} .
$$

We need one more fact: for each $j, c_{i j}$ are simultaneously nonnegative or nonpositive for all $j=1, \ldots, n$. This is proved for $B$ is skew symmetric by Theorem 2.2 . Then, using these facts and the induction hypothesis $C^{\prime T} G^{\prime}=I$, the claim $C^{\prime \prime T} G^{\prime \prime}=I$ can be easily verified.

Remark 4.2. Theorem 4.1 appeared in Kel10b, Pla10a implicitly. Namely, the claim is also a consequence of the following formula in Kel10b, Corollaries 6.9 \& 6.13] and Pla10a, Proposition 3.6]:

$$
g_{i j}^{\prime}=\left[\operatorname{ind}_{T} T_{j}^{\prime}: T_{i}\right]
$$




$$
c_{i j}^{\prime}=\left[\operatorname{ind}_{T^{\prime}}^{\mathrm{op}} T_{i}: T_{j}^{\prime}\right]
$$

where $T=\bigoplus_{i=1}^{n} T_{i}$ and $T^{\prime}=\bigoplus_{i=1}^{n} T_{i}^{\prime}$ are some objects in the 'cluster category' for $\mathcal{A}(B, x, y)$. We ask the reader to consult Pla10a and the forthcoming paper Pla for details.

Now let us turn to the main statement of the section.

For a pair of index sets $I \subset \tilde{I}$, suppose that there is a pair of skew symmetric matrices $B=\left(b_{i j}\right)_{i, j \in I}$ and $\tilde{B}=\left(\tilde{b}_{i j}\right)_{i, j \in \tilde{I}}$ such that $B=\left.\tilde{B}\right|_{I}$ under the restriction of the index set $\tilde{I}$ to $I$. (In terms of quivers, $Q$ is a full subquiver of $\tilde{Q}$.) Then, we say that $B$ is the $I$-restriction of $\tilde{B}$ and $\tilde{B}$ is an $\tilde{I}$-extension of $B$.

Theorem 4.3 (Restriction/Extension Theorem). For $I \subset \tilde{I}$, assume that $B$ and $B^{\prime}$ are skew symmetric matrices such that $B$ is the $I$-restriction of $\tilde{B}$ and $\tilde{B}$ is an $\tilde{I}$-extension of $B$.

(a) (Restriction) Suppose that an $I$-sequence $\mathbf{i}=\left(i_{1}, \ldots, i_{r}\right)$ is a period of $(\tilde{B}, \tilde{x}, \tilde{y})$ in $\mathcal{A}(\tilde{B}, \tilde{x}, \tilde{y})$, Then, $\mathbf{i}$ is also a period of $(B, x, y)$ in $\mathcal{A}(B, x, y)$.

(b) (Extension) Suppose that an $I$-sequence $\mathbf{i}=\left(i_{1}, \ldots, i_{r}\right)$ is a period of $(B, x, y)$ in $\mathcal{A}(B, x, y)$. Then, $\mathbf{i}$ is also a period of $(\tilde{B}, \tilde{x}, \tilde{y})$ in $\mathcal{A}(\tilde{B}, \tilde{x}, \tilde{y})$.

Proof. (a) This is a trivial part. In general, the seed $\left(B^{\prime}, x^{\prime}, y^{\prime}\right)=\mu_{\mathbf{i}}(B, x, y)$ is obtained from the seed $\left(\tilde{B}^{\prime}, \tilde{x}^{\prime}, \tilde{y}^{\prime}\right)=\mu_{\mathbf{i}}(\tilde{B}, \tilde{x}, \tilde{y})$ by restricting the index set $\tilde{I}$ of $\left(\tilde{B}^{\prime}, \tilde{x}^{\prime}, \tilde{y}^{\prime}\right)$ to $I$ and specializing the 'external' initial cluster variables $x_{j}(j \in \tilde{I} \backslash I)$ to 1 appearing in the 'internal' cluster variables $\tilde{x}_{i}^{\prime}(i \in I)$. Thus, the periodicity of $(B, x, y)$ follows from the periodicity of $(\tilde{B}, \tilde{x}, \tilde{y})$.

(b) Set $\left(\tilde{B}^{\prime}, \tilde{x}^{\prime}, \tilde{y}^{\prime}\right):=\mu_{\mathbf{i}}(\tilde{B}, \tilde{x}, \tilde{y})$. Thanks to Theorem 2.6, it is enough to prove

$$
\left[\tilde{y}_{i}^{\prime}\right]_{\mathbf{T}}=\tilde{y}_{i} \quad(i \in \tilde{I}) .
$$

Since the 'external' coefficients $\tilde{y}_{j}^{\prime}(j \in \tilde{I} \backslash I)$ do not influence each other under the mutation $\mu_{\mathbf{i}}$, it is enough to verify (4.7) when there is only one external index $j \in \tilde{I} \backslash I$. Thus, we may assume $I=\{1, \ldots, n\}$ and $\tilde{I}=\{1, \ldots, n+1\}$.

By the periodicity assumption and the exchange relations (2.2) and (2.3), we know a priori the following periodicities hold.

$$
\begin{aligned}
& {\left[\tilde{y}_{i}^{\prime}\right]_{\mathbf{T}}=\tilde{y}_{i} \quad(i \in I),} \\
& \tilde{x}_{n+1}^{\prime}=\tilde{x}_{n+1} .
\end{aligned}
$$

Let us show that (4.8) and (4.9) imply (4.7). We introduce the integers $c_{i j}^{\prime}(i, j \in \tilde{I})$ by

$$
\left[\tilde{y}_{i}^{\prime}\right]_{\mathbf{T}}=\prod_{j=1}^{n+1} \tilde{y}_{j}^{c_{j i}^{\prime}} .
$$

Let $\mathbf{g}_{i}^{\prime}=\left(g_{j i}^{\prime}\right)_{j=1}^{n+1}$ be the $g$-vector for $\tilde{x}_{i}^{\prime}(i \in \tilde{I})$ in (2.5). Then, by Theorem 4.1 the transpose of $C^{\prime}=\left(c_{i j}^{\prime}\right)_{i, j=1}^{n+1}$ and $G^{\prime}=\left(g_{i j}^{\prime}\right)_{i, j=1}^{n+1}$ are inverse to each other, i.e.,

$$
C^{\prime T} G^{\prime}=I \text {. }
$$


Meanwhile, the equalities (4.8) and (4.9) imply that $C^{\prime T}$ and $G^{\prime}$ have the following form:

$$
C^{\prime T}=\left(\begin{array}{ccc|c}
1 & & & 0 \\
& \ddots & & \vdots \\
& & 1 & 0 \\
\hline * & \cdots & * & *
\end{array}\right), \quad G^{\prime}=\left(\begin{array}{ccc|c}
* & \cdots & * & 0 \\
\vdots & & \vdots & \vdots \\
* & \cdots & * & 0 \\
\hline * & \cdots & * & 1
\end{array}\right) .
$$

The condition (4.11) further imposes that the matrix has the form

$$
{C^{\prime}}^{T}=\left(\begin{array}{ccc|c}
1 & & & 0 \\
& \ddots & & \vdots \\
& & 1 & 0 \\
\hline \alpha_{1} & \cdots & \alpha_{n} & 1
\end{array}\right), \quad G^{\prime}=\left(\begin{array}{ccc|c}
1 & & & 0 \\
& \ddots & & \vdots \\
& & 1 & 0 \\
\hline \beta_{1} & \cdots & \beta_{n} & 1
\end{array}\right) .
$$

with $\alpha_{i}=-\beta_{i}$. Now we recall Theorem 2.2 ,

(a) The Laurent monomial (4.10) is either positive or negative. So we have $\alpha_{i} \geq 0$.

(b) For each $i, g_{i j}^{\prime}(j=1, \ldots, n+1)$ are simultaneously nonpositive or nonnegative. So we have $\beta_{i} \geq 0$.

Thus, we have $\alpha_{i}=\beta_{i}=0$ and we conclude that $C^{\prime}=G^{\prime}=I$; therefore, (4.7) holds.

Remark 4.4. The extension theorem (b) was partially formulated by Keller Kela, where only the periodicity of $\tilde{B}$ was considered for the periodicities of seeds for 'pairs of Dynkin diagrams' studied in Kel10b. His result motivates us to formulate Theorem 4.3 (b). A generalized version of the result of Kela will be contained in Pla].

Theorem 4.3 tells that one may assume without losing much generality that the components of a period $\mathbf{i}$ of a seed under study exhaust the index set $I$.

It may be worth to mention that the involution property of a mutation $\mu_{i}$ is also regarded as the simplest case of Extension Theorem applied for the subquiver of type $A_{1}$ consisting of a single vertex $i$.

\section{T-systems and Y-systems}

In this section, we define the T- and Y-systems associated with any period of an exchange matrix. We do it in two steps. First, we treat the special case when the period is 'regular'. The corresponding T- and Y-systems are natural generalizations of the known 'classic' T- and Y-systems. Next, the notions of T- and Y-systems are further extended to the general case. The latter ones will be used in Section 6. We stress that in this section we do not assume the periodicity of seeds. 
5.1. Regular period. Let $B$ a skew symmetrizable matrix with index set $I$.

Definition 5.1. We say that a $\nu$-period $\mathbf{i}$ of $B$ is regular if it is a complete system of representatives of the $\nu$-orbits in $I$; in other words, it satisfies the following conditions:

(A1) All the components of $\mathbf{j}(\mathbf{i}, \nu)=\mathbf{i}|\nu(\mathbf{i})| \cdots \mid \nu^{g-1}(\mathbf{i})$ exhaust $I$, where $g$ is the order of $\nu$.

(A2) All the components of $\mathbf{i}$ belong to distinct $\nu$-orbits in $I$.

Example 5.2. In the previous examples, we have the following regular $\nu$-periods of $B$ or $Q$.

In Example 3.1, $\mathbf{i}_{+} \mid \mathbf{i}_{-}$is a regular period of $B$.

In Example 3.2, $\mathbf{i}_{+}^{\bullet}\left|\mathbf{i}_{+, 1}^{\circ}\right| \mathbf{i}_{-}^{\bullet} \mid \mathbf{i}_{+, 4}^{\circ}$ is a regular $\nu$-period of $Q$.

In Example 3.3. $(1,2)$ is a regular $\rho^{2}$-period of $Q$.

In Example 3.4 (a), $\mathbf{i}_{+} \mid \mathbf{i}_{-}$is a regular period of $Q$.

In Example 3.4 (b), $\mathbf{i}_{+}$is a regular $\nu$-period of $Q$.

In Example 3.5 (a), $\mathbf{i}_{+}$is a regular $\nu$-period of $Q$.

In Example 3.5 (b), $\mathbf{i}_{+}^{\bullet}\left|\mathbf{i}_{+}^{\circ}\right| \mathbf{i}_{-}^{\bullet}$ is a regular $\nu$-period of $Q$.

In Example 3.6, $\mathbf{i}_{+}^{\bullet}|(1)| \mathbf{i}_{-}^{\bullet}$ is a regular $\nu$-period of $Q$.

Suppose that $\mathbf{i}=\left(i_{1}, \ldots, i_{r}\right)$ is a $\nu$-period of $B$. Let us decompose $\mathbf{i}$ into $t$ parts as follows:

$$
\begin{aligned}
\mathbf{i} & =\mathbf{i}(0)|\mathbf{i}(1)| \cdots \mid \mathbf{i}(t-1) \\
\mathbf{i}(p) & =\left(i(p)_{1}, \ldots, i(p)_{r_{p}}\right), \quad \sum_{p=0}^{t-1} r_{p}=r .
\end{aligned}
$$

Let $B(p)(p=0, \ldots, t-1)$ be the matrices defined by the sequence of mutations

$$
B(0)=B \stackrel{\mu_{\mathbf{i}(0)}}{\longrightarrow} B(1) \stackrel{\mu_{\mathbf{i}(1)}}{\longrightarrow} \cdots \stackrel{\mu_{\mathbf{i}(t-1)}}{\longrightarrow} B(t)=\nu(B),
$$

where $\nu(B)=\left(b_{i j}^{\prime}\right)$ is the matrix defined by $b_{\nu(i) \nu(j)}^{\prime}=b_{i j}$.

Definition 5.3. A decomposition (5.1) of a $\nu$-period $\mathbf{i}$ of $B$ is called a slice of $\mathbf{i}$ (of length $t$ ) if it satisfies the following condition:

$$
b(p)_{i(p)_{a}, i(p)_{b}}=0 \quad\left(1 \leq a, b \leq r_{p}\right) \quad \text { for any } p=0, \ldots, t-1 .
$$

For any $\mathbf{i}$, there is at least one slice of $\mathbf{i}$, i.e., the one $\mathbf{i}=\left(i_{1}\right)\left|\left(i_{2}\right)\right| \cdots \mid\left(i_{r}\right)$ of maximal length. In general, there may be several slices of $\mathbf{i}$.

If $\mathbf{i}=\mathbf{i}(0)|\cdots| \mathbf{i}(t-1)$ is a slice of a $\nu$-period of $B$, then, due to Lemma 2.3. each composite mutation $\mu_{\mathbf{i}(p)}$ in (5.3) does not depend on the order of the sequence $\mathbf{i}(p)$ and it is involutive. Furthermore, the sequence (5.3) is extended to 
the infinite one

$$
\begin{aligned}
& \ldots \stackrel{\mu_{\nu}-1(\mathrm{i}(t-2))}{\longleftrightarrow} B(-1) \stackrel{\mu_{\nu}-1(\mathrm{i}(t-1))}{\longleftrightarrow} \\
& B(0) \stackrel{\mu_{\mathrm{i}(0)}}{\longleftrightarrow} B(1) \stackrel{\mu_{\mathrm{i}(1)}}{\longleftrightarrow} \ldots \stackrel{\mu_{\mathrm{i}(t-2)}}{\longleftrightarrow} B(t-1) \stackrel{\mu_{\mathrm{i}(t-1)}}{\longleftrightarrow}
\end{aligned}
$$

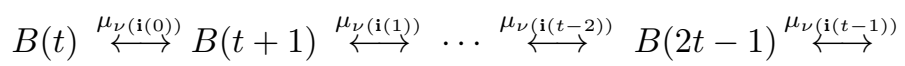

$$
\begin{aligned}
& B(2 t) \stackrel{\mu_{\nu^{2}(\mathbf{i}(0))}^{\longleftrightarrow}}{\longleftrightarrow} \cdots,
\end{aligned}
$$

where $B(n t)=\nu^{n}(B)$ for $n \in \mathbb{Z}$. In particular, $B(g t)=B$, where $g$ is the order of $\nu$. Thus, the sequence (5.5) has a period $g t$ with respect to $u$.

Example 5.4. For the regular $\nu$-periods of $B$ or $Q$ in Examples 5.2, we have the following slices.

In Example 3.1] $\mathbf{i}_{+} \mid \mathbf{i}_{-}$itself is a slice of length 2, and $g t=2$.

In Example 3.2, $\mathbf{i}_{+}^{\bullet}\left|\mathbf{i}_{+, 1}^{\circ}\right| \mathbf{i}_{-}^{\bullet}\left|\mathbf{i}_{+, 4}^{\circ}=\left(\mathbf{i}_{+}^{\bullet} \mid \mathbf{i}_{+, 1}^{\circ}\right)\right|\left(\mathbf{i}_{-}^{\bullet} \mid \mathbf{i}_{+, 4}^{\circ}\right)$ is a slice of length 2, and $g t=10$.

In Example 3.3. $(1,2)=(1) \mid(2)$ is a slice of length 2, and $g t=6$.

In Example 3.4 (a), $\mathbf{i}_{+} \mid \mathbf{i}_{-}$itself is a slice of length 2, and $g t=2$.

In Example 3.4 (b), $\mathbf{i}_{+}$itself is a slice of length 1, and $g t=2$.

In Example 3.5 (a), $\mathbf{i}_{+}$itself is a slice of length 1, and $g t=2$.

In Example 3.5 (b), $\mathbf{i}_{+}^{\bullet}\left|\mathbf{i}_{+}^{\circ}\right| \mathbf{i}_{-}^{\bullet}=\left(\mathbf{i}_{+}^{\bullet} \mid \mathbf{i}_{+}^{\circ}\right) \mid \mathbf{i}_{-}^{\bullet}$ is a slice of length 2 , and $g t=4$.

In Example 3.6. $\mathbf{i}_{+}^{\bullet}|(1)| \mathbf{i}_{-}^{\bullet}=\left(\mathbf{i}_{+}^{\bullet} \mid(1)\right) \mid \mathbf{i}_{-}^{\bullet}$ is a slice of length 2, and $g t=12$.

5.2. T- and $Y$-systems for regular period. Here we introduce the $\mathrm{T}$ - and Y-systems for regular $\nu$-periods, which are especially important in applications. The T- and Y-systems for general $\nu$-periods will be treated in Section 5.5 .

Assume that $\mathbf{i}=\mathbf{i}(0)|\cdots| \mathbf{i}(t-1)$ is a slice of a regular $\nu$-period of $B$.

In view of (5.5), we set $(B(0), x(0), y(0)):=(B, x, y)$ (the initial seed of $\mathcal{A}(B, x, y))$, and consider the corresponding infinite sequence of mutations of seeds

$$
\begin{gathered}
\ldots \stackrel{\mu_{\nu-1}(\mathrm{i}(t-2))}{\longleftrightarrow}(B(-1), x(-1), y(-1)) \stackrel{\mu_{\nu-1} \stackrel{1(\mathrm{i}(t-1))}{\longrightarrow}}{\longrightarrow}(B(0), x(0), y(0)) \stackrel{\mu_{\mathrm{i}(0)}}{\longleftrightarrow} \\
(B(1), x(1), y(1)) \stackrel{\mu_{\mathrm{i}(1)}}{\longleftrightarrow}(B(2), x(2), y(2)) \stackrel{\mu_{\mathrm{i}(2)}}{\longrightarrow} \cdots,
\end{gathered}
$$

thereby introducing a family of clusters $x(u)(u \in \mathbb{Z})$ and coefficients tuples $y(u)$ $(u \in \mathbb{Z})$.

We define a subset $P_{+}$of $I \times \mathbb{Z}$ by $(i, u) \in P_{+}$if and only if $i$ is a component of $\nu^{m}(\mathbf{i}(k))$ for $u=m t+k(m \in \mathbb{Z}, 0 \leq k \leq t-1)$. Plainly speaking, $(i, u) \in P_{+}$ is a forward mutation point in (5.6). Similarly, we define a subset $P_{-}$of $I \times \mathbb{Z}$ by $(i, u) \in P_{-}$if and only if $(i, u-1) \in P_{+}$, namely, $(i, u) \in P_{-}$is a backward mutation point in (5.6). Below we mainly use $P_{+}$. (Alternatively, one may use $P_{-}$ throughout.)

Let $g$ be the order of $\nu$. For each $i \in I$, let $g_{i}$ be the smallest positive integer such that $\nu^{g_{i}}(i)=i$. Therefore, $g_{i}$ is a divisor of $g$. Note that $(i, u) \in P_{+}$if and only if $\left(i, u+t g_{i}\right) \in P_{+}$. It is convenient to define a subset $\tilde{P}_{+}$of $I \times \frac{1}{2} \mathbb{Z}$ by 

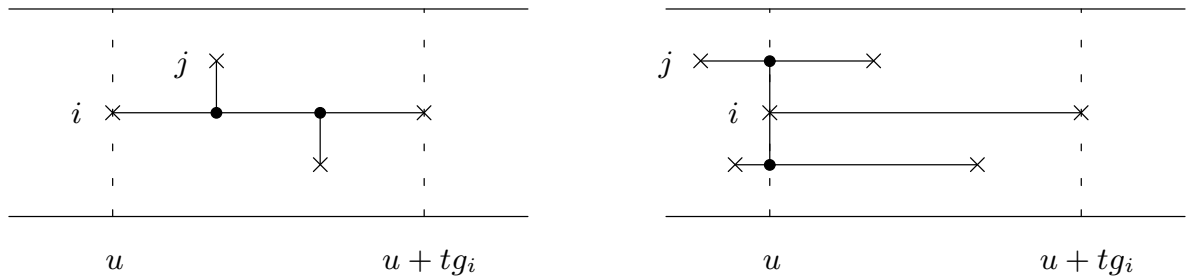

Figure 2. Schematic diagrams for Y-system (left) and T-system (right). A marked point represents a forward mutation point. A vertical bond represents the fact $b_{k i}(v) \neq 0$.

$(i, u) \in \tilde{P}_{+}$if and only if $\left(i, u+\frac{t g_{i}}{2}\right) \in P_{+}$. Consequently, we have

$$
(i, u) \in \tilde{P}_{+} \Longleftrightarrow\left(i, u \pm \frac{t g_{i}}{2}\right) \in P_{+} .
$$

First, we explain what is the Y-system, in short. The sequence of mutations (5.6) gives various relations among coefficients $y_{i}(u)((i, u) \in I \times \mathbb{Z})$ by the exchange relation (2.2). Then, thanks to the assumption (A1) in Definition 5.1, all these coefficients are products of the 'generating' coefficients $y_{i}(u)$ and $1+y_{i}(u)((i, u) \in$ $\left.P_{+}\right)$and their inverses. Here we also used the fact that $y_{i}(u)=y_{i}(u-1)^{-1}$ for $(i, u) \in P_{-}$. Furthermore, these generating coefficients obey some relations, which are the Y-system.

Let us write down the relations explicitly. Take $(i, u) \in P_{+}$and consider the mutation at $(i, u)$, where $y_{i}(u)$ is exchanged to $y_{i}(u+1)=y_{i}(u)^{-1}$. Then, for each $(j, v) \in P_{+}$such that $v \in\left(u, u+t g_{i}\right)$ (i.e., $\left.u<v<u+t g_{i}\right), y_{i}(v)$ are multiplied by factors $\left(1+y_{j}(v)\right)^{-b_{j i}(v)}$ for $b_{j i}(v)<0$ and $\left(1+y_{j}(v)^{-1}\right)^{-b_{j i}(v)}$ for $b_{j i}(v)>0$. The result coincides with the coefficient $y_{i}\left(u+t g_{i}\right)$ by the assumption (A2) in Definition 5.1. See Figure 2 In summary, we have the following relations: For $(i, u) \in P_{+}$,

$$
\begin{aligned}
y_{i}(u) y_{i}\left(u+t g_{i}\right) & =\frac{\prod_{(j, v) \in P_{+}}\left(1+y_{j}(v)\right)^{G_{+}^{\prime}(j, v ; i, u)}}{\prod_{(j, v) \in P_{+}}\left(1+y_{j}(v)^{-1}\right)^{G_{-}^{\prime}(j, v ; i, u)}}, \\
G_{ \pm}^{\prime}(j, v ; i, u) & = \begin{cases}\mp b_{j i}(v) & v \in\left(u, u+t g_{i}\right), b_{j i}(v) \lessgtr 0 \\
0 & \text { otherwise. }\end{cases}
\end{aligned}
$$

Or, equivalently, for $(i, u) \in \tilde{P}_{+}$,

$$
\begin{aligned}
y_{i}\left(u-\frac{t g_{i}}{2}\right) y_{i}\left(u+\frac{t g_{i}}{2}\right) & =\frac{\prod_{(j, v) \in P_{+}}\left(1+y_{j}(v)\right)^{G_{+}(j, v ; i, u)}}{\prod_{(j, v) \in P_{+}}\left(1+y_{j}(v)^{-1}\right)^{G_{-}(j, v ; i, u)}}, \\
G_{ \pm}(j, v ; i, u) & = \begin{cases}\mp b_{j i}(v) & v \in\left(u-\frac{t g_{i}}{2}, u+\frac{t g_{i}}{2}\right), b_{j i}(v) \lessgtr 0 \\
0 & \text { otherwise. }\end{cases}
\end{aligned}
$$


We call the system of relations (5.10) the $Y$-system associated with a slice $\mathbf{i}=$ $\mathbf{i}(0)|\cdots| \mathbf{i}(t-1)$ of a regular $\nu$-period of $B$.

Next, we explain what is the T-system, in short. The sequence of mutations (5.6) gives various relations among cluster variables $x_{i}(u)((i, u) \in I \times \mathbb{Z})$ by the exchange relation (2.3). Again, thanks to the assumption (A1) in Definition 5.1, all these coefficients are represented by the 'generating' cluster variables $x_{i}(u)$ $\left((i, u) \in P_{+}\right)$. Furthermore, these generating cluster variables obey some relations, which are the T-system.

Let us write down the relations explicitly. Take $(i, u) \in P_{+}$and consider the mutation at $(i, u)$. Then, by (2.3) and the assumption (A2) in Definition 5.1, we have

$$
\begin{aligned}
x_{i}(u) x_{i}\left(u+t g_{i}\right)= & \frac{y_{i}(u)}{1+y_{i}(u)} \prod_{(j, v) \in P_{+}} x_{j}(v)^{H_{+}^{\prime}(j, v ; i, u)} \\
& +\frac{1}{1+y_{i}(u)} \prod_{(j, v) \in P_{+}} x_{j}(v)^{H_{-}^{\prime}(j, v ; i, u)}, \\
H_{ \pm}^{\prime}(j, v ; i, u)= & \begin{cases} \pm b_{j i}(u) & u \in\left(v-t g_{j}, v\right), b_{j i}(u) \gtrless 0 \\
0 & \text { otherwise. }\end{cases}
\end{aligned}
$$

See Figure 2, By introducing the 'shifted cluster variables' $\tilde{x}_{i}(u):=x_{i}\left(u+\frac{t g_{i}}{2}\right)$ for $(i, u) \in \tilde{P}_{+}$, these relations can be written in a more 'balanced' form and become parallel to (5.10) as follows: For $(i, u) \in P_{+}$,

$$
\begin{aligned}
\tilde{x}_{i}\left(u-\frac{t g_{i}}{2}\right) \tilde{x}_{i}\left(u+\frac{t g_{i}}{2}\right)= & \frac{y_{i}(u)}{1+y_{i}(u)} \prod_{(j, v) \in \tilde{P}_{+}} \tilde{x}_{j}(v)^{H_{+}(j, v ; i, u)} \\
& +\frac{1}{1+y_{i}(u)} \prod_{(j, v) \in \tilde{P}_{+}} \tilde{x}_{j}(v)^{H_{-}(j, v ; i, u)}, \\
H_{ \pm}(j, v ; i, u)= & \begin{cases} \pm b_{j i}(u) & u \in\left(v-\frac{t g_{j}}{2}, v+\frac{t g_{j}}{2}\right), b_{j i}(u) \gtrless 0 \\
0 & \text { otherwise. }\end{cases}
\end{aligned}
$$

We call the system of relations (5.14) the T-system (with coefficients) associated with a slice $\mathbf{i}=\mathbf{i}(0)|\cdots| \mathbf{i}(t-1)$ of a regular $\nu$-period of $B$.

Let $\mathcal{A}(B, x)$ be the cluster algebra with trivial coefficients with initial seed $(B, x)$. Namely, we set every coefficient to be 1 in the trivial semifield $\mathbf{1}=\{1\}$. Let $\pi_{\mathbf{1}}: \mathbb{P}_{\text {univ }}(y) \rightarrow \mathbf{1}$ be the projection. Let $\left[x_{i}(u)\right]_{\mathbf{1}}$ be the image of $x_{i}(u)$ by the algebra homomorphism $\mathcal{A}(B, x, y) \rightarrow \mathcal{A}(B, x)$ induced from $\pi_{\mathbf{1}}$. By the specialization of (5.14), we have

$$
\left[\tilde{x}_{i}\left(u-\frac{t g_{i}}{2}\right)\right]_{\mathbf{1}}\left[\tilde{x}_{i}\left(u+\frac{t g_{i}}{2}\right)\right]_{\mathbf{1}}=\prod_{(j, v) \in \tilde{P}_{+}}\left[\tilde{x}_{j}(v)\right]_{\mathbf{1}}^{H_{+}(j, v ; i, u)}+\prod_{(j, v) \in \tilde{P}_{+}}\left[\tilde{x}_{j}(v)\right]_{\mathbf{1}}^{H_{-}(j, v ; i, u)}
$$

We call the system of relations (5.16) the T-system (without coefficients) associated with a slice $\mathbf{i}=\mathbf{i}(0)|\cdots| \mathbf{i}(t-1)$ of a regular $\nu$-period of $B$. 
Needless to say, the unbalanced form (5.8) and (5.12) may be also useful in some situation.

Remark 5.5. For a given regular $\nu$-period $\mathbf{i}$, different choices of slices of $\mathbf{i}$ give different Y-systems/T-systems. But they are easily identified by 'change of variables', so the choice of a slice is not essential. (See Proposition 5.8 for a more precise statement.) However, in view of the sequence (5.6), it is economical, and often natural, to use a slice whose length $t$ is minimal among all the other slices, as in Example 5.4.

One may think that the Y- and T-systems are the both sides of the coin in the sense that they directly determine each other as follows.

Proposition 5.6 (Duality). Let $D=\operatorname{diag}\left(d_{i}\right)_{i \in I}$ be a diagonal matrix such that ${ }^{t}(D B)=-D B$. Let $G_{ \pm}(j, v ; i, u)$ and $H_{ \pm}(j, v ; i, u)$ the ones in (5.11) and (5.15), respectively. Then, the following relation holds for any $(j, v) \in P_{+}$and $(i, u) \in \tilde{P}_{+}$.

$$
d_{j} G_{ \pm}(j, v ; i, u)=d_{i} H_{ \pm}(i, u ; j, v) .
$$

In particular, if $B$ is skew symmetric, we have

$$
G_{ \pm}(j, v ; i, u)=H_{ \pm}(i, u ; j, v)
$$

Proof. We recall that any matrix $B^{\prime}$ obtained from $B$ by mutation shares the same diagonalizing matrix $D$ with $B$ [FZ02, Proposition 4.5]. Then, by comparing (5.11) and (5.15), we immediately obtain the claim.

5.3. Examples. Let us write down the Y- and T-systems explicitly for the ones in Example 3.5 .

(a) $(X, \ell)=\left(A_{4}, 4\right)$. Let $Q, \mathbf{i}_{+}$, and $\nu$ be the one therein. Then, $\mathbf{i}_{+}$is a regular $\nu$-period of $Q$. We regard $\mathbf{i}_{+}$as a slice of itself of length 1 , and consider the associated Y- and T-systems. We use the index set $I=\{1,2,3,4\} \times\{1,2,3\}$ such that $(i, j) \in I$ corresponds to the vertex at the $i$ th column (from the left) and the $j$ th row (from the bottom). Thus, we have the data $t=1, g=2$, and $g_{(i, j)}=2$ for any $(i, j) \in I$. The condition for the forward mutation points is given by

$$
((i, j), u) \in P_{+} \Longleftrightarrow i+j+u \text { is even. }
$$

By writing $y_{(i, j)}(u)$ and $\tilde{x}_{(i, j)}(u)$ as $y_{i, j}(u)$ and $\tilde{x}_{i, j}(u)$, the resulting Y-system and T-system (without coefficients) are as follows.

$Y$-system: For $((i, j), u) \in \tilde{P}_{+}$,

$$
y_{i, j}(u-1) y_{i, j}(u+1)=\frac{\left(1+y_{i-1, j}(u)\right)\left(1+y_{i+1, j}(u)\right)}{\left(1+y_{i, j-1}(u)^{-1}\right)\left(1+y_{i, j+1}(u)^{-1}\right)},
$$

where $y_{0, j}(u)=y_{5, j}(u)=0$ and $y_{i, 0}(u)^{-1}=y_{i, 4}(u)^{-1}=0$ in the right hand side.

T-system: For $((i, j), u) \in P_{+}$,

$$
\left[\tilde{x}_{i, j}(u-1)\right]_{\mathbf{1}}\left[\tilde{x}_{i, j}(u+1)\right]_{\mathbf{1}}=\left[\tilde{x}_{i-1, j}(u)\right]_{\mathbf{1}}\left[\tilde{x}_{i+1, j}(u)\right]_{\mathbf{1}}+\left[\tilde{x}_{i, j-1}(u)\right]_{\mathbf{1}}\left[\tilde{x}_{i, j+1}(u)\right]_{\mathbf{1}},
$$


where $\left[\tilde{x}_{0, j}(u)\right]_{\mathbf{1}}=\left[\tilde{x}_{5, j}(u)\right]_{\mathbf{1}}=\left[\tilde{x}_{i, 0}(u)\right]_{\mathbf{1}}=\left[\tilde{x}_{i, 4}(u)\right]_{\mathbf{1}}=1$ in the right hand side.

These are the $\mathrm{Y}$ - and T-systems associated with the quantum affine algebras of type $A_{4}$ with level 4 . The relation (5.21) is also a special case of Hirota's bilinear difference equation Hir77, and it is one of the most studied difference equations in various view points. See $\mathrm{IIK}^{+} 10 \mathrm{c}$ for more information.

(b) $(X, \ell)=\left(B_{4}, 4\right)$. Let $Q, \mathbf{i}_{+}^{\bullet}, \mathbf{i}_{-}^{\bullet}, \mathbf{i}_{+}^{\circ}, \mathbf{i}_{-}^{\circ}$, and $\nu$ be the one therein. Then, $\left(\mathbf{i}_{+}^{\bullet} \mid \mathbf{i}_{+}^{\circ}\right) \mid \mathbf{i}_{-}^{\bullet}$ is a regular $\nu$-period of $Q$. We regard it as a slice of itself of length 2, and consider the associated Y- and T-systems. We use the index set $I$ which is the disjoint union of $\{1,2,3,5,6,7\} \times\{1,2,3\}$ and $\{4\} \times\{1, \ldots, 7\}$ such that $(i, j) \in I$ corresponds to the vertex at the $i$ th column (from the left) and the $j$ th row (from the bottom). Thus, we have the data $t=2, g=2$, and $g_{(i, j)}=2$ for $i \neq 4$ and $g_{(4, j)}=1$. The condition for the forward mutation points is given by

$$
((i, j), u) \in P_{+} \Longleftrightarrow \begin{cases}(i, j) \in \mathbf{i}_{+}^{\circ} \sqcup \mathbf{i}_{+}^{\circ} & u \equiv 0(4) \\ (i, j) \in \mathbf{i}_{-}^{\circ} & u \equiv 1,3(4) \\ (i, j) \in \mathbf{i}_{+}^{\circ} \sqcup \mathbf{i}_{-}^{\circ} & u \equiv 2(4) .\end{cases}
$$

The resulting Y-system and T-system (without coefficients) are as follows, where the 'boundary terms' in the right hand should be ignored as before.

$Y$-system: For $((i, j), u) \in \tilde{P}_{+}$with $i=1,2,6,7$,

$$
y_{i, j}(u-2) y_{i, j}(u+2)=\frac{\left(1+y_{i-1, j}(u)\right)\left(1+y_{i+1, j}(u)\right)}{\left(1+y_{i, j-1}(u)^{-1}\right)\left(1+y_{i, j+1}(u)^{-1}\right)},
$$

and, with $i=3,4,5$,

$$
\begin{aligned}
& y_{3, j}(u-2) y_{3, j}(u+2)=\frac{\left(1+y_{2, j}(u)\right)\left(1+y_{4,2 j-1}(u)\right)\left(1+y_{4,2 j+1}(u)\right)}{\left(1+y_{4,2 j}(u-1)\right)\left(1+y_{4,2 j}(u+1)\right)}, \\
& y_{4,2 j}(u-1) y_{4,2 j}(u+1)= \begin{cases}\frac{1+y_{3, j}(u)}{\left(1+y_{4,2 j-1}(u)^{-1}\right)\left(1+y_{4,2 j+1}(u)^{-1}\right)} & u+2 j \equiv 0(4) \\
\frac{1+y_{5, j}(u)^{2}}{\left(1+y_{4,2 j-1}(u)^{-1}\right)\left(1+y_{4,2 j+1}(u)^{-1}\right)} & u+2 j \equiv 2(4),\end{cases} \\
& y_{4,2 j+1}(u-1) y_{4,2 j+1}(u+1)=\frac{1}{\left(1+y_{4,2 j}(u)^{-1}\right)\left(1+y_{4,2 j+2}(u)^{-1}\right)}, \\
& \left(1+y_{6, j}(u)\right)\left(1+y_{4,2 j-1}(u)\right)\left(1+y_{4,2 j+1}(u)\right) \\
& y_{5, j}(u-2) y_{5, j}(u+2)=\frac{\times\left(1+y_{4,2 j}(u-1)\right)\left(1+y_{4,2 j}(u+1)\right)}{\left(1+y_{5, j-1}(u)^{-1}\right)\left(1+y_{5, j+1}(u)^{-1}\right)} \text {. }
\end{aligned}
$$

T-system: For $((i, j), u) \in P_{+}$with $i=1,2,6,7$,

$$
\left[\tilde{x}_{i, j}(u-2)\right]_{\mathbf{1}}\left[\tilde{x}_{i, j}(u+2)\right]_{\mathbf{1}}=\left[\tilde{x}_{i-1, j}(u)\right]_{\mathbf{1}}\left[\tilde{x}_{i+1, j}(u)\right]_{\mathbf{1}}+\left[\tilde{x}_{i, j-1}(u)\right]_{\mathbf{1}}\left[\tilde{x}_{i, j+1}(u)\right]_{\mathbf{1}},
$$


and, with $i=3,4,5$,

$$
\begin{aligned}
& {\left[\tilde{x}_{3, j}(u-2)\right]_{\mathbf{1}}\left[\tilde{x}_{3, j}(u+2)\right]_{\mathbf{1}}=\left[\tilde{x}_{2, j}(u)\right]_{\mathbf{1}}\left[\tilde{x}_{4,2 j}(u)\right]_{\mathbf{1}}+\left[\tilde{x}_{3, j-1}(u)\right]_{\mathbf{1}}\left[\tilde{x}_{3, j+1}(u)\right]_{\mathbf{1}},} \\
& {\left[\tilde{x}_{4,2 j}(u-1)\right]_{\mathbf{1}}\left[\tilde{x}_{4,2 j}(u+1)\right]_{\mathbf{1}}} \\
& = \begin{cases}{\left[\tilde{x}_{5, j}(u-1)\right]_{\mathbf{1}}\left[\tilde{x}_{3, j}(u+1)\right]_{\mathbf{1}}+\left[\tilde{x}_{4,2 j-1}(u)\right]_{\mathbf{1}}\left[\tilde{x}_{4,2 j+1}(u)\right]_{\mathbf{1}}} & u+2 j \equiv 1(4) \\
{\left[\tilde{x}_{3, j}(u-1)\right]_{\mathbf{1}}\left[\tilde{x}_{5, j}(u+1)\right]_{\mathbf{1}}+\left[\tilde{x}_{4,2 j-1}(u)\right]_{\mathbf{1}}\left[\tilde{x}_{4,2 j+1}(u)\right]_{\mathbf{1}}} & u+2 j \equiv 3(4),\end{cases} \\
& {\left[\tilde{x}_{4,2 j+1}(u-1)\right]_{\mathbf{1}}\left[\tilde{x}_{4,2 j+1}(u+1)\right]_{\mathbf{1}}} \\
& = \begin{cases}{\left[\tilde{x}_{5, j}(u)\right]_{\mathbf{1}}\left[\tilde{x}_{3, j+1}(u)\right]_{\mathbf{1}}+\left[\tilde{x}_{4,2 j}(u)\right]_{\mathbf{1}}\left[\tilde{x}_{4,2 j+2}(u)\right]_{\mathbf{1}}} & u+2 j \equiv 0(4) \\
{\left[\tilde{x}_{3, j}(u)\right]_{\mathbf{1}}\left[\tilde{x}_{5, j+1}(u)\right]_{\mathbf{1}}+\left[\tilde{x}_{4,2 j}(u)\right]_{\mathbf{1}}\left[\tilde{x}_{4,2 j+2}(u)\right]_{\mathbf{1}}} & u+2 j \equiv 2(4),\end{cases} \\
& {\left[\tilde{x}_{5, j}(u-2)\right]_{\mathbf{1}}\left[\tilde{x}_{5, j}(u+2)\right]_{\mathbf{1}}=\left[\tilde{x}_{6, j}(u)\right]_{\mathbf{1}}\left[\tilde{x}_{4,2 j}(u)\right]_{\mathbf{1}}+\left[\tilde{x}_{5, j-1}(u)\right]_{\mathbf{1}}\left[\tilde{x}_{5, j+1}(u)\right]_{\mathbf{1}} \text {. }}
\end{aligned}
$$

Under a suitable identification of variables, they coincide with the Y-and Tsystems associated with the quantum affine algebras of type $B_{4}$ with level 4 . See $\left[\mathrm{IIK}^{+} 10 \mathrm{a}\right.$ for more information.

5.4. Standalone versions of $\mathbf{T}$ - and $\mathbf{Y}$-systems. Here we establish certain formal property concerning T- and Y-systems.

We just introduced the T-systems (without coefficient, for simplicity) (5.16) and $\mathrm{Y}$-systems (5.10) as relations inside a cluster algebra $\mathcal{A}(B, x)$ and a coefficient group $\mathcal{G}(B, y)$. On the other hand, usually these relations appear without their 'ambient' cluster algebras or coefficient groups. Therefore, to apply the cluster algebraic machinery of [FZ07, which has been proved to be so efficient and powerful, it is necessary to establish a precise connection between such 'standalone' Tand Y-systems and the 'built-in' T- and Y-systems inside cluster algebras. In fact, this has been repeatedly established case-by-case for each explicit example (e.g., IIK ${ }^{+} 10 \mathrm{c}$, KNS09, IIK ${ }^{+} 10 \mathrm{a}$, Nak10, NT10]). Here we do it again, and hopeful for the last time, in a general setting.

We continue to use the same notations as in Section 5.2

First, we introduce the ring/group associated with the built-in T-system/Ysystem in cluster algebra/coefficient group.

Definition 5.7. (1) The T-subalgebra $\mathcal{A}_{\mathbf{i}}(B, x)$ of $\mathcal{A}(B, x)$ associated with a slice $\mathbf{i}=\mathbf{i}(0)|\cdots| \mathbf{i}(t-1)$ of a regular $\nu$-period of $B$ is the subring of $\mathcal{A}(B, x)$ generated by $\left[x_{i}(u)\right]_{\mathbf{1}}\left((i, u) \in P_{+}\right)$, or equivalently, generated by $\left[\tilde{x}_{i}(u)\right]_{\mathbf{1}}\left(\left(i, u \in \tilde{P}_{+}\right)\right.$.

(2) The $Y$-subgroup $\mathcal{G}_{\mathbf{i}}(B, y)$ of $\mathcal{G}(B, y)$ associated with a slice $\mathbf{i}=\mathbf{i}(0)|\cdots| \mathbf{i}(t-$ 1) of a regular $\nu$-period of $B$ is the multiplicative subgroup of $\mathcal{G}(B, y)$ generated by $y_{i}(u)$ and $1+y_{i}(u)\left((i, u) \in P_{+}\right)$.

The following fact was casually mentioned in Remark [5.5.

Proposition 5.8. The algebra $\mathcal{A}_{\mathbf{i}}(B, x)$ and the group $\mathcal{G}_{\mathbf{i}}(B, y)$ depend only on $\mathbf{i}$ and do not depend on the choice of a slice of $\mathbf{i}$. 
Proof. Suppose we take two different slices of $\mathbf{i}$. Then, there is a natural bijection between the forward mutation points $(i, u) \leftrightarrow\left(i^{\prime}, u^{\prime}\right)$ for two choices. Then, we have $y_{i}(u)=y_{i^{\prime}}\left(u^{\prime}\right)$ and $x_{i}(u)=x_{i^{\prime}}\left(u^{\prime}\right)$ in $\mathcal{A}(B, x, y)$, thanks to the condition (5.4) and Lemma 2.3

Next, we introduce the corresponding ring/group for the standalone T-system/Ysystem.

Definition 5.9. (1) Let $\mathcal{T}(B, \mathbf{i})$ be the commutative ring over $\mathbb{Z}$ with identity element, with generators $T_{i}(u)^{ \pm 1}\left((i, u) \in \tilde{P}_{+}\right)$and relations (5.16), where $\left[\tilde{x}_{i}(u)\right]_{1}$ is replaced with $T_{i}(u)$, together with $T_{i}(u) T_{i}(u)^{-1}=1$. Let $\mathcal{T}^{\circ}(B, \mathbf{i})$ be the subring of $\mathcal{T}(B, \mathbf{i})$ generated by $T_{i}(u)\left((i, u) \in \tilde{P}_{+}\right)$.

(2) Let $y(B, \mathbf{i})$ be the semifield with generators $Y_{i}(u)\left((i, u) \in P_{+}\right)$and relations (5.10), where $y_{i}(u)$ is replaced with $Y_{i}(u)$. Let $y^{\circ}(B, \mathbf{i})$ be the multiplicative subgroup of $y(B, \mathbf{i})$ generated by $Y_{i}(u)$ and $1+Y_{i}(u)\left((i, u) \in P_{+}\right)$.

Two rings/groups defined above are isomorphic.

Theorem 5.10. (1) The ring $\mathcal{T}^{\circ}(B, \mathbf{i})$ is isomorphic to $\mathcal{A}_{\mathbf{i}}(B, x)$ by the correspondence $T_{i}(u) \mapsto\left[\tilde{x}_{i}(u)\right]_{\mathbf{1}}$.

(2) The group yo $^{\circ}(B, \mathbf{i})$ is isomorphic to $\mathcal{G}_{\mathbf{i}}(B, y)$ by the correspondence $Y_{i}(u) \mapsto$ $y_{i}(u)$ and $1+Y_{i}(u) \mapsto 1+y_{i}(u)$.

Proof. (1) The map $\rho: T_{i}(u) \mapsto\left[\tilde{x}_{i}(u)\right]_{\mathbf{1}}$ is a ring homomorphism by definition. We can construct the inverse of $\rho$ as follows. For each $i \in I$, let $u_{i} \in \mathbb{Z}$ be the smallest nonnegative $u_{i}$ such that $\left(i, u_{i}\right) \in P_{+}$. We define a ring homomorphism $\phi: \mathbb{Z}\left[x_{i}^{ \pm 1}\right]_{i \in I} \rightarrow \mathcal{T}(B, \mathbf{i})$ by $x_{i}^{ \pm 1} \mapsto T_{i}\left(u_{i}-\frac{t g_{i}}{2}\right)^{ \pm 1}$. Thus, we have $\phi:\left[\tilde{x}_{i}\left(u_{i}-\right.\right.$ $\left.\left.\frac{t g_{i}}{2}\right)\right]_{\mathbf{1}}=\left[x_{i}\left(u_{i}\right)\right]_{\mathbf{1}}=\left[x_{i}(0)\right]_{\mathbf{1}} \mapsto T_{i}\left(u_{i}-\frac{t g_{i}}{2}\right)$. Furthermore, one can prove that $\phi:\left[\tilde{x}_{i}(u)\right]_{\mathbf{1}} \mapsto T_{i}(u)$ for any $(i, u) \in \tilde{P}_{+}$by induction on the forward mutations for $u>u_{i}-\frac{t g_{i}}{2}$ and on the backward mutations for $u<u_{i}-\frac{t g_{i}}{2}$, using the common T-systems for the both sides. By the restriction of $\phi$ to $\mathcal{A}_{\mathbf{i}}(B, x)$, we obtain a ring homomorphism $\varphi: \mathcal{A}_{\mathbf{i}}(B, x) \rightarrow \mathcal{T}^{\circ}(B, \mathbf{i})$, which is the inverse of $\rho$.

(2) This is parallel to (1). The map $\rho: Y_{i}(u) \mapsto y_{i}(u), 1+Y_{i}(u) \mapsto 1+y_{i}(u)$ is a group homomorphism by definition. We can construct the inverse of $\rho$ as follows. For each $i \in I$, let $u_{i} \in \mathbb{Z}$ be the largest nonpositive $u_{i}$ such that $\left(i, u_{i}\right) \in P_{+}$. We define a semifield homomorphism $\phi: \mathbb{P}_{\text {univ }}\left(y_{i}\right)_{i \in I} \rightarrow y(B, \mathbf{i})$ as follows. If $u_{i}=0$, then $\phi\left(y_{i}\right)=Y_{i}(0)$. If $u_{i}<0$, we define

$$
\phi\left(y_{i}\right)=Y_{i}\left(u_{i}\right)^{-1} \frac{\prod_{(j, v)}\left(1+Y_{j}(v)\right)^{-b_{j i}(v)}}{\prod_{(j, v)}\left(1+Y_{j}(v)^{-1}\right)^{b_{j i}(v)}},
$$

where the product in the numerator is taken for $(j, v) \in P_{+}$such that $u_{i}<v<0$ and $b_{j i}(v)<0$, and the product in the denominator is taken for $(j, v) \in P_{+}$such that $u_{i}<v<0$ and $b_{j i}(v)>0$. Then, we have $\phi: y_{i}\left(u_{i}\right) \mapsto Y_{i}\left(u_{i}\right)$. Furthermore, one can prove that $\phi: y_{i}(u) \mapsto Y_{i}(u)$ for any $(i, u) \in P_{+}$by induction on the 
forward mutations for $u>u_{i}$ and on the backward mutations for $u<u_{i}$, using the common Y-systems for the both sides. By the restriction of $\phi$ to $\mathcal{G}_{\mathbf{i}}(B, y)$, we obtain a group homomorphism $\varphi: \mathcal{G}_{\mathbf{i}}(B, y) \rightarrow y^{\circ}(B, \mathbf{i})$, which is the inverse of $\rho$.

Aside from the direct connection between T- and Y-systems in Proposition 5.6. there is an algebraic connection, which has been noticed since the inception of the original T- and Y-systems KP92, KNS94.

Proposition 5.11. Let $\mathcal{T}(B, \mathbf{i})$ be the ring in Definition 5.9. For each $(i, u) \in P_{+}$, we set

$$
Y_{i}(u):=\frac{\prod_{(j, v) \in \tilde{P}_{+}} T_{j}(v)^{H_{+}(j, v ; i, u)}}{\prod_{(j, v) \in \tilde{P}_{+}} T_{j}(v)^{H_{-}(j, v ; i, u)}} .
$$

Then, $Y_{i}(u)$ satisfies the $Y$-system (5.10) in $\mathcal{T}(B, \mathbf{i})$ by replacing $y_{i}(u)$ with $Y_{i}(u)$.

Note that (5.28) is the ratio of the first and second terms in (5.16).

Proof. Thanks to the isomorphism in Theorem 5.10, one can work in the localization of $\mathcal{A}_{\mathbf{i}}(B, x)$ by generators $\left[\tilde{x}_{i}(u)\right]_{\mathbf{1}}\left((i, u) \in \tilde{P}_{+}\right)$, which is a subring of the ambient field $\mathbb{Q}(x)$. The claim is translated therein as follows: For each $(i, u) \in P_{+}$, we set

$$
\bar{y}_{i}(u):=\frac{\prod_{(j, v) \in \tilde{P}_{+}}\left[\tilde{x}_{j}(v)\right]_{\mathbf{1}}^{H_{+}(j, v ; i, u)}}{\prod_{(j, v) \in \tilde{P}_{+}}\left[\tilde{x}_{j}(v)\right]_{\mathbf{1}}^{H_{-}(j, v ; i, u)}}=\frac{\prod_{(j, v) \in P_{+}}\left[x_{j}(v)\right]_{\mathbf{1}}^{H_{+}^{\prime}(j, v ; i, u)}}{\prod_{(j, v) \in P_{+}}\left[x_{j}(v)\right]_{\mathbf{1}}^{H_{-}^{\prime}(j, v ; i, u)}}=\prod_{j \in I}\left[x_{j}(u)\right]_{\mathbf{1}}^{b_{j i}(u)} .
$$

Then $\bar{y}_{i}(u)$ satisfies the $Y$-system (5.10) by replacing $y_{i}(u)$ with $\bar{y}_{i}(u)$. In fact, this claim is an immediate consequence of [FZ07, Proposition 3.9].

Remark 5.12. Some classic examples of $\mathrm{T}$ - and $\mathrm{Y}$-systems are not always in the 'straight form' presented here, but represented by generators $T_{\bar{i}}(u)$ and $Y_{\bar{i}}(u)$ whose indices $\bar{i}$ belong to the orbit space $I / \nu$ of $I$ by $\nu$. For example, the T- and Y-systems for type $(X, \ell)=\left(B_{4}, 4\right)$ in Section 5.3 IIK ${ }^{+} 10 \mathrm{a}$, and the sine-Gordon T- and Y-systems for Example 3.6 [NT10] are such cases. In these examples, it is just a 'change of notation' for generators. However, this makes the reconstruction of the initial exchange matrix $B$ from given T- or Y-systems nontrivial, because a priori we only know $I / \nu$, and we have to find out true index set $I$ and $\nu$ with some guesswork. 
5.5. T- and Y-systems for general period. Conceptually, the notions of Tand Y-systems can be straightforwardly extended to general $\nu$-periods of $B$, though they become a little apart from the 'classic' $\mathrm{T}$ - and Y-systems. We will use them in Section 6

Let $\mathbf{i}=\mathbf{i}(0)|\cdots| \mathbf{i}(t-1)$ be a slice of any (not necessarily regular) $\nu$-period $\mathbf{i}$ of $B$. One can still define the sequence of seeds $(B(u), x(u), y(u))(u \in \mathbb{Z})$ and the forward mutation points $(i, u) \in P_{+}$as in the regular case.

Fix $i \in I$, and let

$$
\ldots,(i, u),\left(i, u^{\prime}\right),\left(i, u^{\prime \prime}\right), \ldots \quad\left(\cdots<u<u^{\prime}<u^{\prime \prime}<\ldots\right)
$$

be the sequence of the forward mutation points. (It may be empty for some $i$.) In general, if it is not empty, the sequence $\ldots, u, u^{\prime}, u^{\prime \prime}, \ldots$ is periodic for $u \rightarrow u+t g$, but it does not necessarily have the common difference. For each $(i, u) \in P_{+}$, let $\left(i, u+\lambda_{+}(i, u)\right)$ and $\left(i, u-\lambda_{-}(i, u)\right)$ be the nearest ones to $(i, u)$ in the sequence (5.30) in the forward and backward directions, respectively; in other words, $(i, u-$ $\left.\lambda_{-}(i, u)\right),(i, u),\left(i, u+\lambda_{+}(i, u)\right)$ are three consecutive forward mutation points in (5.30). If $\mathbf{i}$ is regular, then $\lambda_{ \pm}(i, u)=t g_{i}$, which is the common difference (therefore, called regular). In general, we have $0<\lambda_{ \pm}(i, u)<t g, \lambda_{ \pm}(i, u+t g)=$ $\lambda_{ \pm}(i, u)$, and

$$
\lambda_{+}\left(i, u-\lambda_{-}(i, u)\right)=\lambda_{-}(i, u) .
$$

Let $J(\mathbf{i}, \nu)$ be the subset of $I$ consisting of all the components of $\mathbf{j}(\mathbf{i}, \nu)$. Note that the condition (A1) in Definition 5.1 means that $J(\mathbf{i}, \nu)=I$.

Using these notations, the relations (5.8) and (5.12) are generalized as follows. For $(i, u) \in P_{+}$,

$$
\begin{aligned}
y_{i}(u) y_{i}\left(u+\lambda_{+}(i, u)\right) & =\frac{\prod_{(j, v) \in P_{+}}\left(1+y_{j}(v)\right)^{G_{+}^{\prime}(j, v ; i, u)}}{\prod_{(j, v) \in P_{+}}\left(1+y_{j}(v)^{-1}\right)^{G_{-}^{\prime}(j, v ; i, u)},} \\
G_{ \pm}^{\prime}(j, v ; i, u) & = \begin{cases}\mp b_{j i}(v) & v \in\left(u, u+\lambda_{+}(i, u)\right), b_{j i}(v) \lessgtr 0 \\
0 & \text { otherwise, }\end{cases}
\end{aligned}
$$

and

$$
\begin{aligned}
x_{i}(u) x_{i}\left(u+\lambda_{+}(i, u)\right)= & \frac{y_{i}(u)}{1+y_{i}(u)} \prod_{j \in I \backslash J(\mathbf{i}, \nu): b_{j i}>0} x_{j}^{b_{j i}(u)} \prod_{(j, v) \in P_{+}} x_{j}(v)^{H_{+}^{\prime}(j, v ; i, u)} \\
& +\frac{1}{1+y_{i}(u)} \prod_{j \in I \backslash J(\mathbf{i}, \nu): b_{j i}<0} x_{j}^{-b_{j i}(u)} \prod_{(j, v) \in P_{+}} x_{j}(v)^{H_{-}^{\prime}(j, v ; i, u)}, \\
H_{ \pm}^{\prime}(j, v ; i, u)= & \begin{cases} \pm b_{j i}(u) & u \in\left(v-\lambda_{-}(j, v), v\right), b_{j i}(u) \gtrless 0 \\
0 & \text { otherwise. }\end{cases}
\end{aligned}
$$


When a $\nu$-period $\mathbf{i}$ of $B$ satisfies the condition (A1) in Definition [5.1, the relation (5.34) slightly simplifies as

$$
\begin{aligned}
x_{i}(u) x_{i}\left(u+\lambda_{+}(i, u)\right)= & \frac{y_{i}(u)}{1+y_{i}(u)} \prod_{(j, v) \in P_{+}} x_{j}(v)^{H_{+}^{\prime}(j, v ; i, u)} \\
& +\frac{1}{1+y_{i}(u)} \prod_{(j, v) \in P_{+}} x_{j}(v)^{H_{-}^{\prime}(j, v ; i, u)} .
\end{aligned}
$$

Unfortunately, one cannot simultaneously rewrite (5.32) and (5.34) / (5.36) into the balanced form similar to (5.10) and (5.14). Therefore, we just regard (5.32) and (5.34) /(5.36) as the Y-and T-systems associated with a slice $\mathbf{i}=\mathbf{i}(0)|\cdots| \mathbf{i}(t-1)$ of a $\nu$-period of $B$.

Accordingly, Proposition 5.6 is generalized as follows.

Proposition 5.13 (Duality). Let $D=\operatorname{diag}\left(d_{i}\right)_{i \in I}$ be a diagonal matrix such that ${ }^{t}(D B)=-D B$. Let $G_{ \pm}^{\prime}(j, v ; i, u)$ and $H_{ \pm}^{\prime}(j, v ; i, u)$ the ones in (5.33) and (5.35), respectively. Then, the following relation holds for any $(j, v) \in P_{+}$and $(i, u) \in \tilde{P}_{+}$.

$$
d_{j} G_{ \pm}^{\prime}\left(j, v ; i, u-\lambda_{-}(i, u)\right)=d_{i} H_{ \pm}^{\prime}(i, u ; j, v) .
$$

In particular, if $B$ is skew symmetric, we have

$$
G_{ \pm}^{\prime}\left(j, v ; i, u-\lambda_{-}(i, u)\right)=H_{ \pm}^{\prime}(i, u ; j, v) .
$$

Proof. This is immediate from (5.33) and (5.35).

The results in Section 5.4 can be also generalized and/or modified straightforwardly. We leave it as an exercise for the reader.

\section{Dilogarithm identities}

Now we are ready to work on the main subject of the paper, the dilogarithm identities associated with any period of a seed. They are natural generalizations of the results in the former examples FS95, Cha05, Nak09b, IIK ${ }^{+} 10 \mathrm{a}, \mathrm{IIK}^{+} 10 \mathrm{~b}$, NT10]. The subject has originated from the pioneering works by Bazhanov, Kirillov, and Reshetikhin [KR86, Kir89, KR90, BR90]. See also Kir95, Zag07, Nah07 for further background of the identities.

6.1. Dilogarithm identities. Here we concentrate on the case when the exchange matrix $B$ is skew symmetric. (See Section 6.5 for the skew symmetrizable case.) Let $\mathbf{i}$ be any period of $(B, x, y)$. In particular, $\mathbf{i}$ is also a period of $B$. Let $\mathbf{i}=\mathbf{i}(0)|\cdots| \mathbf{i}(t-1)$ be any slice of $\mathbf{i}$. Then, the sequence of seeds $(B(u), x(u), y(u))$ $(u \in \mathbb{Z})$ and the forward mutation points $(i, u) \in P_{+}$are defined as in Section 5. and we have the associated Y-system (5.32). 
To emphasize the periodicity, we set $\Omega:=t$. Then, by the periodicity assumption, we have

$$
y_{i}(u+\Omega)=y_{i}(u)
$$

Accordingly, we define the 'fundamental regions' $S_{+}$of forward mutation points by

$$
S_{+}=\left\{(i, u) \in P_{+} \mid 0 \leq u<\Omega\right\} .
$$

Thanks to Theorem 2.2 each Laurent monomial $\left[y_{i}(u)\right]_{\mathbf{T}}$ in $y\left((i, u) \in P_{+}\right)$is either positive or negative. Let $N_{+}$(resp. $N_{-}$) be the total number of positive (resp. negative) monomials $\left[y_{i}(u)\right]_{\mathbf{T}}$ in the fundamental region $S_{+}$.

Let $L(x)$ be the Rogers dilogarithm function [Lew81].

$$
L(x)=-\frac{1}{2} \int_{0}^{x}\left\{\frac{\log (1-y)}{y}+\frac{\log y}{1-y}\right\} d y \quad(0 \leq x \leq 1) .
$$

It satisfies the following properties.

$$
\begin{gathered}
L(0)=0, \quad L(1)=\frac{\pi^{2}}{6}, \\
L(x)+L(1-x)=\frac{\pi^{2}}{6} \quad(0 \leq x \leq 1) .
\end{gathered}
$$

The following is the main result of the paper.

Theorem 6.1 (Dilogarithm identity in the skew symmetric case). Suppose that a family of real positive numbers $\left\{Y_{i}(u) \mid(i, u) \in P_{+}\right\}$satisfies the $Y$-system (5.32) by replacing $y_{i}(u)$ with $Y_{i}(u)$. Then, we have the identities.

$$
\begin{aligned}
\frac{6}{\pi^{2}} \sum_{(i, u) \in S_{+}} L\left(\frac{Y_{i}(u)}{1+Y_{i}(u)}\right) & =N_{-}, \\
\frac{6}{\pi^{2}} \sum_{(i, u) \in S_{+}} L\left(\frac{1}{1+Y_{i}(u)}\right) & =N_{+} .
\end{aligned}
$$

Two identities (6.6) and (6.7) are equivalent to each other due to (6.5).

6.2. Proof of Theorem 6.1. We prove Theorem 6.1 using the method developed by FS95, Cha05, Nak09b.

We start from a very general theorem on the 'constancy property' of dilogarithm sum by Frenkel-Szenes [FS95]. For any multiplicative abelian group $A$, let $A \otimes_{\mathbb{Z}} A$ be the additive abelian group with generators $f \otimes g(f, g \in A)$ and relations

$$
(f g) \otimes h=f \otimes h+g \otimes h, \quad f \otimes(g h)=f \otimes g+f \otimes h .
$$

It follows that we have

$$
1 \otimes h=h \otimes 1=0, \quad f^{-1} \otimes g=f \otimes g^{-1}=-f \otimes g .
$$


Let $S^{2} A$ be the subgroup of $A \otimes_{\mathbb{Z}} A$ generated by $f \otimes f(f \in A)$. Define $\bigwedge^{2} A$ be the quotient group of $A \otimes_{\mathbb{Z}} A$ by $S^{2} A$. In $A \otimes_{\mathbb{Z}} A$ we use $\wedge$ instead of $\otimes$. Hence, $f \wedge g=-g \wedge f$ holds.

Now let $\mathcal{I}$ be any open or closed interval of $\mathbb{R}$, and let $\mathcal{C}(\mathcal{I})$ be the multiplicative abelian group of all the differentiable functions $f(t)$ from $\mathcal{I}$ to the set of all the positive real numbers $\mathbb{R}_{+}$.

Theorem 6.2 ([FS95, Proposition 1]). Let $f_{1}(t), \ldots, f_{k}(t)$ be differentiable functions from $\mathcal{I}$ to $(0,1)$. Suppose that they satisfy the following relation in $\bigwedge^{2} \mathcal{C}(\mathcal{I})$.

$$
\sum_{i=1}^{k} f_{i}(t) \wedge\left(1-f_{i}(t)\right)=0 \quad \text { (constancy condition) }
$$

Then, the dilogarithm sum $\sum_{i=1}^{k} L\left(f_{i}(t)\right)$ is constant with respect to $t \in \mathcal{I}$.

We remark that the proof of the theorem by [FS95] is quite simple, just showing the derivative of the dilogarithm sum vanishes by the symmetry reason.

Next we use the idea of Chapoton [Cha05] to integrate the condition (6.10) in the cluster algebra setting. We are going to prove the following claim.

Proposition 6.3. In $\bigwedge^{2} \mathbb{P}_{\text {univ }}(y)$, the following relation holds.

$$
\sum_{(i, u) \in S_{+}} \frac{y_{i}(u)}{1+y_{i}(u)} \wedge \frac{1}{1+y_{i}(u)}=0,
$$

or, equivalently,

$$
\sum_{(i, u) \in S_{+}} y_{i}(u) \wedge\left(1+y_{i}(u)\right)=0 .
$$

It is clear that the relations (6.11) and (6.12) are equivalent. We call them the constancy condition for the dilogarithm identities (6.6) and (6.7). Temporarily assuming Proposition 6.3. we prove the following theorem, which is equivalent to Theorem 6.1.

Theorem 6.4. Let $\mathbb{R}_{+}$be the semifield of all the positive real numbers with the usual multiplication and addition. Then, for any semifield homomorphism $\varphi$ : $\mathbb{P}_{\text {univ }}(y) \rightarrow \mathbb{R}_{+}$, we have the identity,

$$
\begin{aligned}
& \frac{6}{\pi^{2}} \sum_{(i, u) \in S_{+}} L\left(\varphi\left(\frac{y_{i}(u)}{1+y_{i}(u)}\right)\right)=N_{-}, \\
& \frac{6}{\pi^{2}} \sum_{(i, u) \in S_{+}} L\left(\varphi\left(\frac{1}{1+y_{i}(u)}\right)\right)=N_{+} .
\end{aligned}
$$

Proof. We concentrate on the first case (6.13). The proof separates into two steps. 
Step 1: Consistency. Let us first show that the left hand side of (6.13) is independent of the choice of $\varphi$. Note that any semifield homomorphism $\varphi$ is determined by the values $\varphi\left(y_{i}\right)(i \in I)$ of generators of $\mathbb{P}_{\text {univ }}(y)$. Let $\varphi_{0}$ and $\varphi_{1}$ be different homomorphisms from $\mathbb{P}_{\text {univ }}(y)$ to $\mathbb{R}_{+}$. Then we introduce the one parameter family of homomorphisms $\varphi_{t}: \mathbb{P}_{\text {univ }}(y) \rightarrow \mathbb{R}_{+}(t \in[0,1])$ interpolating $\varphi_{0}$ to $\varphi_{1}$ by $\varphi_{t}\left(y_{i}\right)=(1-t) \varphi_{0}\left(y_{i}\right)+t \varphi_{1}\left(y_{i}\right)$. Set $f_{i, u}(t):=\varphi_{t}\left(y_{i}(u) /\left(1+y_{i}(u)\right)\right)$ $\left((i, u) \in S_{+}\right)$and regard them as a family of functions of $t$ in the interval $[0,1]$. Then, due to (6.11), they satisfy the constancy condition (6.10) with $\mathcal{I}=[0,1]$. Then, by Theorem 6.2 the left hand side of (6.13) for $\varphi=\varphi_{t}$ is independent of $t$; in particular, it is the same for $\varphi=\varphi_{0}$ and $\varphi_{1}$.

Step 2: Evaluation at $0 / \infty$ limit. Since we established the constancy of the left hand side of (6.13), we evaluate it at a certain limit of $\varphi$ where each value $\varphi\left(y_{i}(u) /\left(1+y_{i}(u)\right)\right)\left((i, u) \in S_{+}\right)$goes to either 0 or $\infty(0 / \infty$ limit $)$. Then, by (6.4), the value of the left hand side of (6.13) is the total number of $(i, u) \in S_{+}$ such that the value $\varphi\left(y_{i}(u) /\left(1+y_{i}(u)\right)\right)$ goes to $\infty$. It is not obvious that such a limit exists. However, simply take the one parameter family of the homomorphism $\varphi_{t}: \mathbb{P}_{\text {univ }} \rightarrow \mathbb{R}_{+}(t \in(0, \varepsilon))$ for some $\varepsilon>0$ defined by $\varphi_{t}\left(y_{i}\right)=t$ for any $i \in I$. Then, thanks to (2.6) and the parts (a) and (c) of Conjecture 2.1/Theorem 2.2.

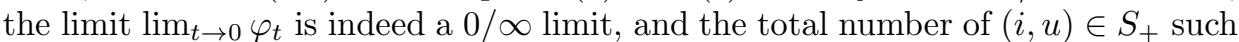
that the value $\varphi\left(y_{i}(u) /\left(1+y_{i}(u)\right)\right)$ goes to $\infty$ is exactly $N_{-}$.

Remark 6.5. In the above proof, Step 1 (constancy) is due to [FS95], and Step 2 (evaluation at $0 / \infty$ limit) is due to Cha05].

6.3. First proof of Proposition 6.3. Now we only have to prove Proposition 6.3. We give two proofs in this and the next subsections. The first one here is a generalization of the former proofs for special cases $\mathrm{Nak09b}$, IIK ${ }^{+} 10 \mathrm{a}$, which is a somewhat brute force proof with 'change of indices'.

To start, we claim that one can assume all the components of $\mathbf{i}$ exhaust $I$. In fact, if the condition does not hold, one may reduce the index set $I$ so that the condition holds, since doing that does not affect the Y-system (5.32). Note that the condition is nothing but the condition (A1) in Definition 5.1 with $\nu=\mathrm{id}$. Thus, the accompanying T-system has the simplified form (5.36). Furthermore, by the periodicity assumption and Theorem 4.3 (a), we have

$$
x_{i}(u+\Omega)=x_{i}(u) .
$$

Now, let $F_{i}(u)$ be the $F$-polynomials at $(i, u)$.

Lemma 6.6. The following properties hold.

(a) Periodicity: $F_{i}(u+\Omega)=F_{i}(u)$.

(b) For $(i, u) \in S_{+}$,

$$
\begin{aligned}
F_{i}(u) F_{i}\left(u+\lambda_{+}(i, u)\right)= & {\left[\frac{y_{i}(u)}{1+y_{i}(u)}\right] \prod_{\mathbf{T}} F_{j, v) \in P_{+}}(v)^{H_{+}^{\prime}(j, v ; i, u)} } \\
& +\left[\frac{1}{1+y_{i}(u)}\right]_{\mathbf{T}} \prod_{(j, v) \in P_{+}} F_{j}(v)^{H_{-}^{\prime}(j, v ; i, u)} .
\end{aligned}
$$


(c) For $(i, u) \in S_{+}$,

$$
\begin{gathered}
y_{i}(u)=\left[y_{i}(u)\right]_{\mathbf{T}} \frac{\prod_{(j, v) \in P_{+}} F_{j}(v)^{H_{+}^{\prime}(j, v ; i, u)}}{\prod_{(j, v) \in P_{+}} F_{j}(v)^{H_{-}^{\prime}(j, v ; i, u)}}, \\
1+y_{i}(u)=\left[1+y_{i}(u)\right]_{\mathbf{T}} \frac{F_{i}(u) F_{i}\left(u+\lambda_{+}(i, u)\right)}{\prod_{(j, v) \in P_{+}} F_{j}(v)^{H_{-}^{\prime}(j, v ; i, u)}} .
\end{gathered}
$$

Proof. (a) This is obtained from the specialization of 6.15). (b) This is obtained from the specialization of (5.36). (c) The first equality is obtained by rewriting (2.6) with (5.35). The second one is obtained from the first one and (b).

Proof of Proposition 6.3. We prove (6.12). We put (6.17) and (6.18) into the left hand side of (6.12), expand it, then, sum them up into three parts as follows.

The first part consists of the terms only involving tropical coefficients, i.e.,

$$
\sum_{(i, u) \in S_{+}}\left[y_{i}(u)\right]_{\mathbf{T}} \wedge\left[1+y_{i}(u)\right]_{\mathbf{T}} .
$$

By Theorem 2.2. each monomial $\left[y_{i}(u)\right]_{\mathbf{T}}$ is either positive or negative. If it is positive, then $\left[y_{i}(u)\right]_{\mathbf{T}} \wedge\left[1+y_{i}(u)\right]_{\mathbf{T}}=\left[y_{i}(u)\right]_{\mathbf{T}} \wedge 1=0$. If it is negative, then, $\left[y_{i}(u)\right]_{\mathbf{T}} \wedge\left[1+y_{i}(u)\right]_{\mathbf{T}}=\left[y_{i}(u)\right]_{\mathbf{T}} \wedge\left[y_{i}(u)\right]_{\mathbf{T}}=0$. Therefore, the sum 6.19$)$ vanishes.

The second part consists of the terms involving both tropical coefficients and $F$-polynomials. We separate them into five parts,

$$
\begin{gathered}
\sum_{(i, u) \in S_{+}}\left[y_{i}(u)\right]_{\mathrm{T}} \wedge F_{i}(u), \\
\sum_{(i, u) \in S_{+}}\left[y_{i}(u)\right]_{\mathrm{T}} \wedge F_{i}\left(u+\lambda_{+}(i, u)\right)=\sum_{(i, u) \in S_{+}}\left[y_{i}\left(u-\lambda_{-}(i, u)\right)\right]_{\mathrm{T}} \wedge F_{i}(u), \\
-\sum_{(i, u) \in S_{+}}\left[y_{i}(u)\right]_{\mathrm{T}} \wedge \prod_{(j, v) \in P_{+}} F_{j}(v)^{H_{-}^{\prime}(j, v ; i, u)} \\
=\sum_{\sum_{(i, u) \in S_{+}}} \prod_{(1, u) \in S_{+}(j, v) \in P_{+}}\left[y_{j}(v)\right]_{\mathrm{T}}^{-H_{-}^{\prime}(i, u ; j, v)} \wedge F_{i}(u), \\
\left.\sum_{(i, u) \in S_{+}}\left[1+y_{i}(u)\right]_{\mathrm{T}} \wedge \prod_{(j, v) \in P_{+}} F_{j}(v)^{H_{+}^{\prime}(j, v ; i, u)}\right]_{\mathrm{T}} \wedge \prod_{(j, v) \in P_{+}} F_{j}(v)^{H_{-}^{\prime}(j, v ; i, u)} \\
\sum_{(i, u) \in S_{+}(j, v) \in P_{+}}\left[1+y_{j}(v)\right]_{\mathrm{T}}^{-H_{+}^{\prime}(i, u ; j, v)} \wedge F_{i}(u), \\
\sum_{(i, u) \in S_{+}(j, v) \in P_{+}}\left[1+y_{j}(v)\right]_{\mathrm{T}}^{H_{-}^{\prime}(i, u ; j, v)} \wedge F_{i}(u),
\end{gathered}
$$


where we changed indices and also used the periodicity of $\left[y_{i}(u)\right]_{\mathbf{T}}, F_{i}(u), \lambda_{-}(i, u)$, and $H_{ \pm}^{\prime}(j, v ; i, u)$. Recall the relation $H_{ \pm}^{\prime}(i, u ; j, v)=G_{ \pm}^{\prime}\left(j, v ; i, u-\lambda_{-}(i, u)\right)$ in (5.38). Then, the sum of the above five terms vanishes due to the 'tropical Ysystem'

$$
\left[y_{i}\left(u-\lambda_{-}(i, u)\right)\right]_{\mathbf{T}}\left[y_{i}(u)\right]_{\mathbf{T}}=\frac{\prod_{(j, v) \in P_{+}}\left[1+y_{j}(v)\right]_{\mathbf{T}}^{G_{+}^{\prime}\left(j, v ; i, u-\lambda_{-}(i, u)\right)}}{\prod_{(j, v) \in P_{+}}\left[1+y_{j}(v)^{-1}\right]_{\mathbf{T}}^{G_{-}^{\prime}\left(j, v ; i, u-\lambda_{-}(i, u)\right)}},
$$

which is a specialization of (5.32).

The third part consists of the terms involving only $F$-polynomials. It turns out that this part requires the most elaborated treatment. We separate them into three parts,

$$
\begin{gathered}
(\mathrm{A})=\sum_{(i, u) \in S_{+}} \frac{\prod_{(j, v) \in P_{+}} F_{j}(v)^{H_{+}^{\prime}(j, v ; i, u)}}{\prod_{(j, v) \in P_{+}} F_{j}(v)^{H_{-}^{\prime}(j, v ; i, u)}} \wedge F_{i}(u), \\
(\mathrm{B})=\sum_{(i, u) \in S_{+}} \frac{\prod_{(j, v) \in P_{+}} F_{j}(v)^{H_{+}^{\prime}(j, v ; i, u)}}{\prod_{(j, v) \in P_{+}} F_{j}(v)^{H_{-}^{\prime}(j, v ; i, u)}} \wedge F_{i}\left(u+\lambda_{+}(i, u)\right), \\
(\mathrm{C})=\sum_{(i, u) \in S_{+}} \prod_{(j, v) \in P_{+}} F_{j}(v)^{H_{+}^{\prime}(j, v ; i, u)} \wedge \prod_{(j, v) \in P_{+}} F_{j}(v)^{-H_{-}^{\prime}(j, v ; i, u) .}
\end{gathered}
$$

Let us rewrite each term so that their cancellation becomes manifest.

The first term (A) is rewritten as follows.

$$
\begin{aligned}
(\mathrm{A}) & =\sum_{(i, u) \in S_{+}} \prod_{\substack{(j, v) \in P_{+} \\
u \in\left(v-\lambda_{-}(j, v), v\right)}} F_{j}(v)^{b_{j i}(u)} \wedge F_{i}(u) \\
= & \sum_{\substack{(i, u) \in S_{+},(j, v) \in P_{+} \\
u \in\left(v-\lambda_{-}(j, v), v\right)}} b_{j i}(u) F_{j}(v) \wedge F_{i}(u) \\
= & \sum_{\substack{(i, u) \in S_{+},(j, v) \in P_{+} \\
v \in\left(u-\lambda_{-}(i, u), u\right)}} b_{j i}(v) F_{j}(v) \wedge F_{i}(u) \\
= & \frac{1}{2} \sum_{\substack{(i, u) \in S_{+},(j, v) \in P_{+} \\
\left(u-\lambda_{-}(i, u), u\right) \cap\left(v-\lambda_{-}(j, v), v\right) \neq \emptyset}} b_{j i}(\min (u, v)) F_{j}(v) \wedge F_{i}(u) .
\end{aligned}
$$

Here the third line is obtained from the second one by the exchange $(i, u) \leftrightarrow(j, v)$ of indices, the skew symmetric property $b_{j i}(u)=-b_{i j}(u)$, and the periodicity; the 
last line is obtained by averaging the second and the third ones; therefore, there is the factor $1 / 2$ in the front. We also mention that, in the last line, the pair $(i, u),(j, v)$ with $u=v$ does not contribute to the sum, because in that case we have $b_{j i}(u)=0$ due to the condition (5.4).

Similarly, the second term (B) is rewritten as follows.

$$
\begin{aligned}
& (\mathrm{B})=\sum_{(i, u) \in S_{+}} \prod_{\substack{(j, v) \in P_{+} \\
u \in\left(v-\lambda_{-}(j, v), v\right)}} F_{j}(v)^{b_{j i}(u)} \wedge F_{i}\left(u+\lambda_{+}(i, u)\right) \\
& =\sum_{\substack{(i, u) \in S_{+},(j, v) \in P_{+} \\
u-\lambda_{-}(i, u) \in\left(v-\lambda_{-}(j, v), v\right)}} b_{j i}\left(u-\lambda_{-}(i, u)\right) F_{j}(v) \wedge F_{i}(u) \\
& =\frac{1}{2} \sum_{\substack{(i, u) \in S_{+},(j, v) \in P_{+} \\
\left(u-\lambda_{-}(i, u), u\right) \cap\left(v-\lambda_{-}(j, v), v\right) \neq \emptyset}} b_{j i}\left(\max \left(u-\lambda_{-}(i, u), v-\lambda_{-}(j, v)\right)\right) F_{j}(v) \wedge F_{i}(u) .
\end{aligned}
$$

The third term $(\mathrm{C})$ is written as follows.

$$
\begin{aligned}
& (\mathrm{C})=\sum_{(i, u),(j, v) \in P_{+}}\left(\sum_{\begin{array}{c}
(k, w) \in S_{+} \\
k \in\left(u-\lambda_{-}(i, u), u\right) \cap\left(v-\lambda_{-}(j, v), v\right) \\
b_{j k}(w)>0, b_{i k}(w)<0
\end{array}} b_{j k}(w) b_{i k}(w)\right) F_{j}(v) \wedge F_{i}(u) \\
& =\sum_{(i, u),(j, v) \in P_{+}}\left(\sum_{\begin{array}{c}
(k, w) \in S_{+} \\
k \in\left(u-\lambda_{-}(i, u), u\right) \cap\left(v-\lambda_{-}(j, v), v\right) \\
b_{j k}(w)<0, b_{i k}(w)>0
\end{array}}-b_{j k}(w) b_{i k}(w)\right) F_{j}(v) \wedge F_{i}(u) \\
& =\frac{1}{2} \sum_{(i, u) \in S_{+},(j, v) \in P_{+}}\left(\sum_{\substack{(k, w) \in P_{+} \\
k \in\left(u-\lambda_{-}(i, u), u\right) \cap\left(v-\lambda_{-}(j, v), v\right)}}\right. \\
& \left.-\frac{1}{2}\left(b_{j k}(w)\left|b_{k i}(w)\right|+\left|b_{j k}(w)\right| b_{k i}(w)\right)\right) F_{j}(v) \wedge F_{i}(u) .
\end{aligned}
$$

Here the second line is obtained from the first one by the exchange $(i, u) \leftrightarrow(j, v)$ of indices; the last line is obtained by averaging the first and the second ones; therefore, there is the factor $1 / 2$ in the front. We also mention that, in the last line, $(k, w)$ with $b_{j k}(w)$ and $b_{i k}(w)$ having the same sign does not contribute to the second sum, because in that case $b_{j k}(w)\left|b_{k i}(w)\right|+\left|b_{j k}(w)\right| b_{k i}(w)=0$.

Now the sum $(\mathrm{A})+(\mathrm{B})+(\mathrm{C})$ cancel due to the mutation of matrices (see (2.1))

$$
\begin{aligned}
& b_{j i}(\min (u, v))=-b_{j i}\left(\max \left(u-\lambda_{i}(i, u), v-\lambda_{j}(j, v)\right)\right) \\
& +\sum_{\substack{(k, w) \in P_{+} \\
k \in\left(u-\lambda_{-}(i, u), u\right) \cap\left(v-\lambda_{-}(j, v), v\right)}} \frac{1}{2}\left(b_{j k}(w)\left|b_{k i}(w)\right|+\left|b_{j k}(w)\right| b_{k i}(w)\right)
\end{aligned}
$$

for $\left(u-\lambda_{-}(i, u), u\right) \cap\left(v-\lambda_{-}(j, v), v\right) \neq \emptyset$. 
6.4. Local version of constancy condition and second proof of Proposition 6.3 . Here we give a 'local' version of Proposition 6.3, thereby providing an alternative proof of Proposition 6.3. The formula here is parallel to the ones in [FG09a, Lemma 6.1] and [FG09b, Lemma 2.17] by Fock and Goncharov. In fact, we have reached the formula while trying to interpolate Proposition 6.3 and their results. An important difference from their formulas is the use of $F$-polynomials in 6.26).

Let $\mathcal{A}(B, x, y)$ be any cluster algebra. For each seed $\left(B^{\prime}, x^{\prime}, y^{\prime}\right)$, we set

$$
V^{\prime}:=\frac{1}{2} \sum_{i \in I} F_{i}^{\prime} \wedge\left(y_{i}^{\prime}\left[y_{i}^{\prime}\right]_{\mathbf{T}}\right)=\sum_{i \in I} F_{i}^{\prime} \wedge y_{i}^{\prime}+\frac{1}{2} \sum_{i, j \in I} b_{i j}^{\prime} F_{i}^{\prime} \wedge F_{j}^{\prime},
$$

where $F_{i}^{\prime}=F_{i}^{\prime}(y)(i \in I)$ are the $F$-polynomials for $\left(B^{\prime}, x^{\prime}, y^{\prime}\right)$.

Proposition 6.7 (Local constancy, cf. [FG09a, Lemma 6.1], [FG09b, Proposition 2.14 \& Lemma 2.17]). Let $\left(B^{\prime}, x^{\prime}, y^{\prime}\right)$ and $\left(B^{\prime \prime}, x^{\prime \prime}, y^{\prime \prime}\right)$ be any seeds such that $\left(B^{\prime \prime}, x^{\prime \prime}, y^{\prime \prime}\right)=\mu_{k}\left(B^{\prime}, x^{\prime}, y^{\prime}\right)$. Then, we have the following relation in $\bigwedge^{2} \mathbb{P}_{\text {univ }}(y)$.

$$
V^{\prime \prime}-V^{\prime}=y_{k}^{\prime} \wedge\left(1+y_{k}^{\prime}\right) .
$$

Proof. Our proof is parallel to the first proof of Proposition 6.3. We use the following property of the corresponding $F$-polynomials, which are derived as Lemma 6.6.

(a) For the above $k$,

$$
F_{k}^{\prime} F_{k}^{\prime \prime}=\left[\frac{y_{k}^{\prime}}{1+y_{k}^{\prime}}\right]_{\mathbf{T}} \prod_{j: b_{j k}^{\prime}>0} F_{j}^{\prime b_{j k}^{\prime}}+\left[\frac{1}{1+y_{k}^{\prime}}\right]_{\mathbf{T}} \prod_{j: b_{j k}^{\prime}<0} F_{j}^{\prime-b_{j k}^{\prime}}
$$

(b) For any $i \in I$,

$$
y_{i}^{\prime}=\left[y_{i}^{\prime}\right]_{\mathbf{T}} \frac{\prod_{j: b_{j i}^{\prime}>0} F_{j}^{\prime b_{j i}^{\prime}}}{\prod_{j: b_{j i}^{\prime}<0} F_{j}^{\prime-b_{j i}^{\prime}}}=\left[y_{i}^{\prime}\right]_{\mathbf{T}} \prod_{j \in I} F_{j}^{\prime b_{j i}^{\prime}} .
$$

(c) For the above $k$,

$$
1+y_{k}^{\prime}=\left[1+y_{k}^{\prime}\right]_{\mathbf{T}} \frac{F_{k}^{\prime} F_{k}^{\prime \prime}}{\prod_{j: b_{j k}^{\prime}<0} F_{j}^{\prime-b_{j k}^{\prime}}}
$$

We put (6.29) and (6.30) into the right hand side of (6.27), expand it, then, sum them up into three parts as before.

The first part consists of the single term $\left[y_{k}^{\prime}\right]_{\mathbf{T}} \wedge\left[1+y_{k}^{\prime}\right]_{\mathbf{T}}$, which vanishes by the same reason as before. 
The second part consists of the terms involving both tropical coefficients and $F$-polynomials. We separate them into five parts as (6.20). By an easy calculation as before, they are summarized as follows.

$$
\sum_{i \in I} F_{i}^{\prime \prime} \wedge\left[y_{i}^{\prime \prime}\right]_{\mathbf{T}}-\sum_{i \in I} F_{i}^{\prime} \wedge\left[y_{i}^{\prime}\right]_{\mathbf{T}}
$$

The third part consists of the terms involving only $F$-polynomials. We separate them into three parts as (6.22)-6.24). Then, they are summarized as follows.

$$
\frac{1}{2} \sum_{i \in I} F_{i}^{\prime \prime} \wedge \frac{y_{i}^{\prime \prime}}{\left[y_{i}^{\prime \prime}\right]_{\mathbf{T}}}-\frac{1}{2} \sum_{i \in I} F_{i}^{\prime} \wedge \frac{y_{i}^{\prime}}{\left[y_{i}^{\prime}\right]_{\mathbf{T}}}
$$

Therefore, we obtain the claim.

Proposition 6.3 is immediately obtained from Proposition 6.7 as follows.

For any $u \in \mathbb{Z}$, we set

$$
V(u)=\frac{1}{2} \sum_{i \in I} F_{i}(u) \wedge\left(y_{i}(u)\left[y_{i}(u)\right]_{\mathbf{T}}\right) .
$$

Note that $V(0)=0$ because $F_{i}(0)=1$. Then, by Proposition 6.7, we have, for $u>0$

$$
V(u)=\sum_{\substack{(k, v) \in P_{+} \\ 0 \leq v<u}} y_{k}(v) \wedge\left(1+y_{k}(v)\right) .
$$

Then, from the periodicity $V(\Omega)=V(0)=0$, we obtain (6.12).

6.5. Skew symmetrizable case. For a skew symmetrizable matrix $B$, the dilogarithm identities should be slightly modified.

Let $D=\operatorname{diag}\left(d_{i}\right)_{i \in I}$ be the (left) skew symmetrizer of $B$, namely, $d_{i} b_{i j}=$ $-d_{j} b_{j i}$ holds. Let $d=\operatorname{LCM}\left(d_{i}\right)_{i \in I}$ and define $\tilde{d}_{i}=d / d_{i} \in \mathbb{N}(i \in I)$. Then, $\tilde{D}=\operatorname{diag}\left(\tilde{d}_{i}\right)_{i \in I}$ be the right skew symmetrizer of $B$, namely, $b_{i j} \tilde{d}_{j}=-b_{j i} \tilde{d}_{i}$ holds. Let $\tilde{N}_{+}$(resp. $\tilde{N}_{-}$) be the total number of positive (resp. negative) monomials $\left[y_{i}(u)\right]_{\mathbf{T}}$ in the fundamental region $S_{+}$with multiplicity $\tilde{d}_{i}$. Then, the identities (6.6) and (6.7) should be modified as

$$
\begin{gathered}
\frac{6}{\pi^{2}} \sum_{(i, u) \in S_{+}} \tilde{d}_{i} L\left(\varphi\left(\frac{y_{i}(u)}{1+y_{i}(u)}\right)\right)=\tilde{N}_{-}, \\
\frac{6}{\pi^{2}} \sum_{(i, u) \in S_{+}} \tilde{d}_{i} L\left(\varphi\left(\frac{1}{1+y_{i}(u)}\right)\right)=\tilde{N}_{+},
\end{gathered}
$$

and (6.26) and (6.27) should be modified as

$$
V^{\prime}:=\frac{1}{2} \sum_{i \in I} \tilde{d}_{i} F_{i}^{\prime} \wedge\left(y_{i}^{\prime}\left[y_{i}^{\prime}\right]_{\mathbf{T}}\right)=\sum_{i \in I} \tilde{d}_{i} F_{i}^{\prime} \wedge y_{i}^{\prime}+\frac{1}{2} \sum_{i, j \in I} b_{i j}^{\prime} \tilde{d}_{j} F_{i}^{\prime} \wedge F_{j}^{\prime},
$$




$$
V^{\prime \prime}-V^{\prime}=\tilde{d}_{k} y_{k}^{\prime} \wedge\left(1+y_{k}^{\prime}\right) .
$$

Unfortunately, for skew symmetrizable matrices one cannot yet prove (6.35), (6.36), and (6.38). This is because Conjecture 2.1 (the facts (a) and (c), in particular) is not yet proved, and we need it for the existence of the $0 / \infty$ limit and also for $\left[y_{k}^{\prime}\right]_{\mathbf{T}} \wedge\left[1+y_{k}^{\prime}\right]_{\mathbf{T}}$ to vanish. However, one can prove the rest by repeating the same argument. To summarize, we have the following intermediate result in the skew symmetrizable case.

Theorem 6.8. Suppose that Conjecture 2.1 is true. Then, for any skew symmetrizable matrix, we have (6.38) and the identities (6.35) and (6.36).

6.6. Concluding remark. Let us conclude the paper by raising one natural question.

Problem 6.9. Describe the numbers $N_{ \pm}$and $N_{ \pm}^{\prime}$ in Theorems 6.1 and 6.8 by some other ways.

\section{References}

$\left[\mathrm{BMR}^{+} 06\right]$ A. Buan, R. Marsh, M. Reineke, I. Reiten, and G. Todorov, Tilting theory and cluster combinatorics, Adv. in Math. 204 (2006), 572-618; arXiv:math/0402054 [math.RT].

[BR90] V. V. Bazhanov and N. Reshetikhin, Restricted solid-on-solid models connected with simply laced algebras and conformal field theory, J. Phys. A: Math. Gen. 23 (1990), 1477-1492.

[CC06] P. Caldero and F. Chapoton, Cluster algebras as Hall algebras of quiver representations, Comment. Math. Helv. 81 (2006), 595-616; arXiv:math/0410187 [math.RT].

[Cha05] F. Chapoton, Functional identities for the Rogers dilogarithm associated to cluster Y-systems, Bull. London Math. Soc. 37 (2005), 755-760.

[DK09] P. Di Francesco and R. Kedem, Q-systems as cluster algebras II: Cartan matrix of finite type and the polynomial property, Lett. Math. Phys. 89 (2009), 183-216; arXiv:0803.0362 [math.RT].

[DWZ10] H. Derksen, J. Weyman, and A. Zelevinsky, Quivers with potentials and their representations II: Applications to cluster algebras, J. Amer. Math. Soc. 23 (2010), 749-790; arXiv:0904.0676 [math.RA].

[FG07] V. V. Fock and A. B. Goncharov, Dual Teichmüller and lamination spaces, Handbook of Teichüller theory, Vol. I, Eur. Math. Soc., 2007, pp. 647-684, arXiv:math/0510312 [math.DG].

[FG09a] Cluster ensembles, quantization and the dilogarithm, Annales Sci. de l'École Norm. Sup. 42 (2009), 865-930; arXiv:math/0311245 [math.AG].

[FG09b] The quantum dilogarithm and representations of quantum cluster varieties, Invent. Math. 172 (2009), 223-286; arXiv:math/0702397 [math.QA]. 
[FHH01] B. Feng, A. Hanany, and Y-H. He, D-brane gauge theories from toric singularities and toric duality, Nucl. Phys. B595 (2001), 165-200; arXiv:hep-th/0003085

[FM09] A. P. Fordy and R. J. Marsh, Cluster mutation-periodic quivers and associated Laurent sequences, 2009, arXiv:0904.0200 [math.CO].

[FS95] E. Frenkel and A. Szenes, Thermodynamic Bethe ansatz and dilogarithm identities. I, Math. Res. Lett. 2 (1995), 677-693; arXiv:hep-th/9506215.

[FZ02] S. Fomin and A. Zelevinsky, Cluster algebras I. Foundations, J. Amer. Math. Soc. 15 (2002), 497-529 (electronic); arXiv:math/0104151 [math.RT].

[FZ03a] S. Fomin and A. Zelevinsky, Cluster algebras II. Finite type classification, Invent. Math. 154 (2003), 63-121; arXiv:math/0208229 [math.RA].

[FZ03b] Y Y-systems and generalized associahedra, Ann. of Math. 158 (2003), 977-1018; arXiv:hep-th/0111053.

[FZ07] Cluster algebras IV. Coefficients, Compositio Mathematica 143 (2007), 112-164; arXiv:math/0602259 [math.RT].

[GLS09] C. Geiss, B. Leclerc, and J. Schröer, Cluster algebra structures and semicanonical bases for unipotent groups, 2009, arXiv:math/0703039 [math.RT].

[GSV05] M. Gekhtman, M. Shapiro, and A. Vainshtein, Cluster algebras and WeilPetersson forms, Duke Math. J. 127 (2005), 291-311; arXiv:math/0309138 [math.QA].

[GT95] F. Gliozzi and R. Tateo, ADE functional dilogarithm identities and integrable models, Phys. Lett. B348 (1995), 677-693; arXiv:hep-th/9411203

[Her07] D. Hernandez, Drinfeld coproduct, quantum fusion tensor category and applications, Proc. London Math. Soc. 95 (2007), 567-608; arXiv:math/0504269 [math.QA].

[Hir77] R. Hirota, Nonlinear partial difference equations II: Discrete time Toda equations, J. Phys. Soc. Japan 43 (1977), 2074-2078.

[HL10] D. Hernandez and B. Leclerc, Cluster algebras and quantum affine algebras, Duke Math. J. 154 (2010), 265-341, arXiv:0903.1452 [math.QA].

[HW90] H. C. Hutchins and H. J. Weinert, Homomorphisms and kernels of semifields, Periodica Math. Hung. 21 (1990), 113-152.

$\left[\mathrm{IIK}^{+} 10 \mathrm{a}\right]$ R. Inoue, O. Iyama, B. Keller, A. Kuniba, and T. Nakanishi, Periodicities of $T$ and $Y$-systems, dilogarithm identities, and cluster algebras $I$ : Type $B_{r}$, 2010, arXiv:1001.1880 [math.QA].

$\left[\mathrm{IIK}^{+} 10 \mathrm{~b}\right]$, Periodicities of $T$ and $Y$-systems, dilogarithm identities, and cluster algebras II: Types $C_{r}, F_{4}$, and $G_{2}, 2010$, arXiv:1001.1881 [math.QA].

$\left[\mathrm{IIK}^{+} 10 \mathrm{c}\right]$ R. Inoue, O. Iyama, A. Kuniba, T. Nakanishi, and J. Suzuki, Periodicities of $T$ and $Y$-systems, Nagoya Math. J. 197 (2010), 59-174; arXiv:0812.0667 [math.QA].

[Kela] B. Keller, Cluster algebras, cluster categories and periodicity, talk presented at Workshop on Representation theory of algebras 2008, Shizuoka, September, 2008.

[Kelb] B. Keller, Quiver mutation in Java, Java applet available at http://people.math.jussieu.fr/ keller/quivermutation/. 
[Kel10a] B. Keller, Cluster algebras, quiver representations and triangulated categories, Triangulated categories (T. Holm, P. Jørgensen, and R. Rouquier, eds.), Lecture Note Series, vol. 375, London Mathematical Society, Cambridge University Press, 2010, pp. 76-160; arXiv:0807.1960 [math.RT].

[Kel10b] , The periodicity conjecture for pairs of Dynkin diagrams, 2010, arXiv:1001.1531 [math.RT].

[Kir89] A. N. Kirillov, Identities for the Rogers dilogarithm function connected with simple Lie algebras, J. Sov. Math. 47 (1989), 2450-2458.

[Kir95] _ Dilogarithm identities, Prog. Theor. Phys. Suppl. 118 (1995), 61142; arXiv:hep-th/9408113

[KN92] A. Kuniba and T. Nakanishi, Spectra in conformal field theories from the Rogers dilogarithm, Mod. Phys. Lett. A7 (1992), 3487-3494; arXiv:hep-th/9206034

[KNS94] A. Kuniba, T. Nakanishi, and J. Suzuki, Functional relations in solvable lattice models: I. Functional relations and representation theory, Int. J. Mod. Phys. A9 (1994), 5215-5266; arXiv:hep-th/9309137

[KNS09] _ T-systems and Y-systems for quantum affinizations of quantum KacMoody algebras, SIGMA 5 (2009), 108, 23 pages; arXiv:0909.4618 [math.QA].

[KP92] A. Klümper and P. A. Pearce, Conformal weights of RSOS lattice models and their fusion hierarchies, Physica A 183 (1992), 304-350.

[KR86] A. N. Kirillov and N. Yu. Reshetikhin, Exact solution of the Heisenberg XXZ model of spin s, J. Sov. Math. 35 (1986), 2627-2643.

[KR90] A. N. Kirillov and N. Yu. Reshetikhin, Representations of Yangians and multiplicities of the inclusion of the irreducible components of the tensor product of representations of simple Lie algebras,, J. Sov. Math. 52 (1990), 3156-3164.

[Kun93] A. Kuniba, Thermodynamics of the $U_{q}\left(X_{r}^{(1)}\right)$ Bethe ansatz system with $q$ a root of unity, Nucl. Phys. B389 (1993), 209-244.

[Lew81] L. Lewin, Polylogarithms and associated functions, North-Holland, Amsterdam, 1981.

[Nag10] K. Nagao, Donaldson-Thomas theory and cluster algebras, 2010, arXiv:1002.4884 [math.AG].

[Nah07] W. Nahm, Conformal field theory and torsion elements of the Bloch group, Frontiers in Number Theory, Physics, and Geometry II, Springer, Berlin, Heidelberg, 2007, pp. 3-65, arXiv:hep-th/0404120

[Nak09a] H. Nakajima, Quiver varieties and cluster algebras, 2009, arXiv:0905.0002.

[Nak09b] T. Nakanishi, Dilogarithm identities for conformal field theories and cluster algebras: Simply laced case, 2009, arXiv:math.0909.5480 [math.QA], to appear in Nagoya Math. J.

[Nak10] T. Nakanishi, T-systems and Y-systems, and cluster algebras: Tamely laced case, 2010, arXiv:1003.1180 [math.QA].

[NT10] T. Nakanishi and R. Tateo, Dilogarithm identities for sine-Gordon and reduced sine-Gordon Y-systems, 2010, arXiv:1005.4199 [math.QA].

[Pla] P. Plamondon, Calabi-Yau reduction for cluster categories with infinitedimensional morphism spaces, in preparation. 
[Pla10a]_ Cluster algebras via cluster categories with infinite-dimensional morphism spaces, 2010, arXiv:1004.0830 [math.RT].

[Pla10b] Cluster characters for cluster categories with infinite-dimensional morphism spaces, 2010, arXiv:1002.4956 [math.RT].

[RTV93] R. Ravanini, R. Tateo, and A. Valleriani, Dynkin TBA's, Int. J. Mod. Phys. A8 (1993), 1707-1727; arXiv:hep-th/9207040.

[Zag07] D. Zagier, The dilogarithm function, Frontiers in Number Theory, Physics, and Geometry II, Springer, Berlin, Heidelberg, 2007, pp. 3-65.

[Zam91] Al. B. Zamolodchikov, On the thermodynamic Bethe ansatz equations for reflectionless ADE scattering theories, Phys. Lett. B253 (1991), 391-394.

Tomoki Nakanishi, Graduate School of Mathematics, Nagoya University, Nagoya, 4648604, Japan

E-mail: nakanisi@math.nagoya-u.ac.jp 